\title{
DOMAIN DECOMPOSITION WITH NONMATCHING GRIDS: AUGMENTED LAGRANGIAN APPROACH
}

\author{
PATRICK LE TALLEC AND TAOUFIK SASSI
}

\begin{abstract}
We propose and study a domain decomposition method which treats the constraint of displacement continuity at the interfaces by augmented Lagrangian techniques and solves the resulting problem by a parallel version of the Peaceman-Rachford algorithm. We prove that this algorithm is equivalent to the fictitious overlapping method introduced by P.L. Lions. We also prove its linear convergence independently of the discretization step $h$, even if the finite element grids do not match at the interfaces. A new preconditioner using fictitious overlapping and well adapted to three-dimensional elasticity problems is also introduced and is validated on several numerical examples.
\end{abstract}

\section{INTRODUCTION}

In this paper, we are interested in the numerical solution of a second-order linear elliptic problem by a nonoverlapping domain decomposition technique. The model problem under consideration takes the standard form :

Find $u \in V_{0}$ such that

$$
\sum_{i}\left\{a_{i}(u, v)-L_{i}(v)\right\}=0, \quad \forall v \in V_{0},
$$

where $V_{0}$ is the usual Sobolev space

$$
V_{0}=\left\{v \in H^{1}(\Omega), v=0 \text { on } \partial \Omega_{D}\right\},
$$

defined over a given domain $\Omega=\bigcup_{i} \Omega_{i}$ of $\mathbb{R}^{\mathrm{dim}}$. When dealing with a basic Poisson equation, the local forms $a_{i}(u, v)$ (bilinear) and $L_{i}(v)$ are given on each subdomain $\Omega_{i}$ by

$$
\begin{gathered}
a_{i}(u, v)=\int_{\Omega_{i}} \nabla u \cdot \nabla v d x \\
L_{i}(v)=\int_{\Omega_{i}} f \cdot v d x+\int_{\partial \Omega_{N} \cap \partial \Omega_{i}} g \cdot v d a .
\end{gathered}
$$

For more complex linear elasticity problems, we would have instead

$$
a_{i}(u, v)=\int_{\Omega_{i}} \sigma(x, \nabla u): \nabla v d x,
$$

Received by the editor June 2, 1993 and, in revised form, August 3, 1994.

1991 Mathematics Subject Classification. Primary 65N30, 65M55, 65F10, 73G05. 


$$
\sigma(x, \nabla u)=A(x)\left(\nabla u+\nabla^{t} u\right) / 2,
$$

with $A(x)$ a symmetric positive fourth-order elasticity tensor.

In any case, even if $\Omega$ is partitioned into nonoverlapping subdomains $\Omega_{i}$ (Figure 1), problem (1) does not reduce to independent subproblems posed on each subdomain $\Omega_{i}$ because elements of the space $V_{0}$ are constrained to be continuous across the different interfaces $\partial \Omega_{i} \cap \partial \Omega_{j}$. Most nonoverlapping domain decomposition techniques handle this constraint by iterative substructuring methods, which reduce the original problem to an interface problem whose unknown is the trace of $u$ on the interface, and which is solved iteratively by a preconditioned conjugate gradient method (see Bramble, Pasciak and Schatz [4, 5], Dryja, Smith and Widlund [9] and Le Tallec [17] for more details).

The purpose of this paper is to propose and study another numerical strategy which treats the constraint of displacement continuity at the interfaces by a Lagrange multiplier method. Based on augmented Lagrangian techniques, it first rewrites the original global minimization problem as a saddle-point problem and then solves it by a standard saddle-point algorithm which only involves the solution of local subproblems. This turns out to be equivalent to the fictitious overlapping method introduced in [20] and can be proved to converge linearly independently of the discretization step $h$, even if the finite element grids do not match at the interfaces.

A key point in this algorithm is the choice of the scalar product to be used on the interface.

Three different choices will be investigated, both from a mathematical and numerical point of view :

- the $L^{2}$ scalar product, which is the simplest but which leads to an $h$ dependent algorithm,

- a $\Delta^{-1 / 2}$ scalar product, easy to implement in $2 \mathrm{D}$ problems with straight interfaces,

- a new preconditioner using fictitious overlapping and well adapted to threedimensional elasticity problems.

The paper is organized as follows. The continuous problem, the basic Lagrangian formulation and algorithm are introduced in $\S 2$. Convergence results are derived in $\S 3$ for the continuous problem and in $\S 4$ for its Finite Element approximation. A new preconditioner is defined in $\S 5$, and the paper concludes by several three-dimensional numerical calculations, which illustrate the performance of the proposed method and compare them with those obtained by iterative substructuring techniques.

\section{LAGRANGIAN APPROACH OF THE CONTINUOUS PROBLEM}

2.1. Notation. For simplicity, the domain $\Omega$ is decomposed into two nonoverlapping subdomains $\Omega_{i}$ with interface $S$. We now introduce the boundaries (see Figure 1)

$$
\begin{aligned}
\partial \Omega & =\partial \Omega_{D} \cup \partial \Omega_{N}, & & \text { external Dirichlet and Neumann boundaries, } \\
\partial \Omega_{D_{i}} & =\partial \Omega_{D} \cap \partial \Omega_{i}, & & \text { local Dirichlet boundary, }
\end{aligned}
$$




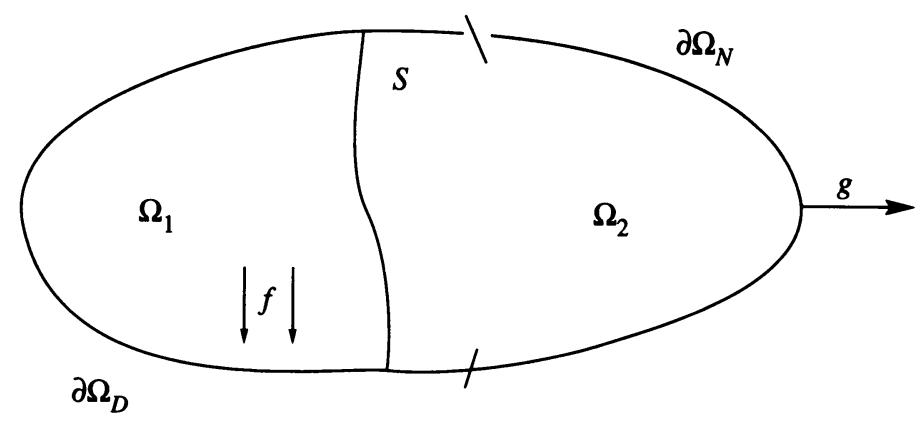

Figure 1. The physical problem

together with the spaces

$$
\begin{aligned}
& V_{i}=\left\{v \in H^{1}\left(\Omega_{i}\right), v=0 \text { on } \partial \Omega_{D_{i}}\right\}, \\
& V=V_{1} \times V_{2} \quad \text { with norm }\|\cdot\|_{V}=\left(\sum_{i}\|\cdot\|_{V_{i}}^{2}\right)^{1 / 2}, \\
& V_{0}=\left\{\left(v_{1}, v_{2}\right) \in V, \operatorname{Tr} v_{1}\left|S=\operatorname{Tr} v_{2}\right|_{S}\right\} \\
& W=\operatorname{Tr} V_{1}\left|S=\operatorname{Tr} V_{2}\right| S .
\end{aligned}
$$

2.2. Lagrangian formulation. Without the linear constraint appearing in the definition of $V_{0}$, we would be faced with two independent problems posed on $\Omega_{1}$ and $\Omega_{2}$, respectively. To preserve this splitting property, a natural idea is then to treat the constraints $\left.v_{1}\right|_{S}=\left.v_{2}\right|_{S}$ by augmented Lagrangian techniques (Fortin and Glowinski [12]), that is, by penalization (one adds a term $\frac{r}{2}\left\|v_{i}-q\right\|_{H^{\frac{1}{2}}(S)}^{2}$ to the energy) and by dualization (one introduces Lagrange multipliers $\lambda_{i}$ of the linear constraints $\left.\left.v_{1}\right|_{S}=\left.v_{2}\right|_{S}=q\right)$.

For this purpose, we introduce:

- an arbitrary scalar product on the interface space $W$ (tentatively equivalent to the $H^{\frac{1}{2}}(S)$ scalar product) given by

$$
(q, \hat{q})=\langle\mathscr{S} q, \hat{q}\rangle, \quad \forall q \in W,
$$

with $\mathscr{S}$ a given positive selfadjoint operator defined from $W$ into $W^{\prime}$, and $\langle.,$.$\rangle the corresponding duality pairing between H^{\frac{1}{2}}(S)$ and its dual,

- the augmented Lagrangian

(3) $\mathscr{L}_{r}(v, q, \lambda)=\sum_{i=1}^{2}\left\{\frac{1}{2} a_{i}\left(u_{i}, v_{i}\right)-L_{i}\left(v_{i}\right)+\frac{r}{2}\left\|v_{i}-q\right\|^{2}+\left(\lambda_{i}, v_{i}-q\right)\right\}$

with $r$ a given arbitrary strictly positive number.

With the notation (2)-(3), we also introduce the transformed problem: 
Find $(u, q, \lambda) \in V \times W \times H$ such that

$$
\left\{\begin{array}{rll}
\text { (i) } \frac{\partial \mathscr{L}_{r}}{\partial v}(u, q, \lambda) \cdot w=0, & \forall w \in V=V_{1} \times V_{2}, \\
\text { (ii) }\left\langle\frac{\partial \mathscr{L}_{r}}{\partial q}(u, q, \lambda), d q\right\rangle=0, & \forall d q \in W=\operatorname{Tr} V, \\
\text { (iii) }\left\langle\frac{\partial \mathscr{L}_{L}}{\partial \lambda}(u, q, \lambda), d \lambda\right\rangle=0, & \forall d \lambda \in H=W^{2},
\end{array}\right.
$$

or in more details:

- equation in $u$

$$
\begin{gathered}
\sum_{i=1}^{2}\left\{a_{i}\left(u_{i}, w_{i}\right)-L_{i}\left(w_{i}\right)+r\left\langle\mathscr{S}\left(u_{i}-q\right), w_{i}\right\rangle+\left\langle\mathscr{S} \lambda_{i}, w_{i}\right\rangle\right\}=0, \\
\forall w \in V=V_{1} \times V_{2},
\end{gathered}
$$

- equation in $q$

$$
\sum_{i=1}^{2}\left\{-r\left\langle\mathscr{S}\left(u_{i}-q\right), d q\right\rangle-\left\langle\mathscr{S} \lambda_{i}, d q\right\rangle\right\}=0, \quad \forall d q \in W=\operatorname{Tr} V_{i},
$$

- equation in $\lambda$

$$
\sum_{i=1}^{2}\left\langle\mathscr{S}\left(u_{i}-q\right), d \lambda_{i}\right\rangle=0 \quad, \quad \forall d \lambda \in H=W^{2} .
$$

Remark 2.1. All the techniques introduced in this paper can be extended to a multidomain partition of $\Omega$ into $\Omega=\bigcup_{i} \Omega_{i}$ with interfaces $S=\bigcup_{i<j} \partial \Omega_{i} \cap$ $\partial \Omega_{j}=\bigcup_{i<j} S_{i j}$. In this case, the global space $V_{0}$ and trace space $W$ would be

$$
\begin{gathered}
V_{0}=\left\{\left(v_{i}\right)_{i} \in \prod_{i} V_{i}, \quad \operatorname{Tr} v_{i}=\operatorname{Tr} v_{j} \text { on } S_{i j}, \forall i<j\right\}, \\
W=\prod_{i<j} W_{i j}, \quad W_{i j}=\operatorname{Tr} V_{i}\left|S_{i j}=\operatorname{Tr} V_{j}\right| S_{i j} .
\end{gathered}
$$

In such a treatment of interfaces, edges and corners are neglected. This is legitimate, both at the continuous and the finite element level, if there are no edges or corners (partition in strips) or if the interfaces are discretized by mortar elements (§4.2) which define discrete traces $\operatorname{Tr}_{i h}$ on faces and not on corners. This treatment can also be extended to general conforming partitions simply by considering any given edge separating, say, four subdomains $\Omega_{i}, \Omega_{j}, \Omega_{k}, \Omega_{l}$ as three distinct faces $S_{i j}, S_{j k}$ and $S_{k l}$.

Remark 2.2. In what follows, unless explicitly stated, the space $W$ is endowed with the norm

$$
\|w\|_{W}=\|\operatorname{Ext}(w)\|_{H^{1 / 2}\left(S \cup \partial \Omega_{D}\right)}
$$

with $\operatorname{Ext}(w)$ the function which is equal to $w$ on $S$ and which is equal to zero on $\partial \Omega_{D}$. With this choice of norm, the trace is a continuous surjection from $V_{i}$ onto $W$. We will refer to this norm $\|\cdot\|_{W}$ and to the associated scalar product as the $H^{1 / 2}(S)$ norm and scalar product. Strictly speaking, this terminology is correct only if the distance between $\partial \Omega_{D}$ and $S$ is strictly positive. 
Remark 2.3. The simplest choice for $\mathscr{S}$ consists in choosing

$$
(q, \hat{q})=\langle\mathscr{S} q, \hat{q}\rangle=\int_{S} q \hat{q} d x .
$$

Unfortunately, this $L^{2}$ scalar product is not equivalent to the $H^{1 / 2}$ scalar product and this has some negative effects on the convergence of the algorithms. Another choice is to use $\mathscr{S}=\left(-\Delta_{S}\right)^{1 / 2}$, where $-\Delta_{S}$ stands for the Laplace Beltrami operator on the interface $S$. For this choice, the scalar product

$$
(q, \hat{q})=\left\langle\left(-\triangle_{S}\right)^{1 / 2} q, \hat{q}\right\rangle
$$

is equivalent to the $H^{1 / 2}$ scalar product. We recall that for a straight face $S$ perpendicular to $0 x_{3}$, we have

$$
\begin{gathered}
\triangle_{S}(q)=\frac{\partial^{2} q}{\partial x_{1}^{2}}+\frac{\partial^{2} q}{\partial x_{2}^{2}} \\
\left(-\triangle_{S}\right)^{1 / 2}(q)=\sum_{j} \sqrt{-\lambda_{j}}\left(\int_{S} q(x) e_{j}(x) d x\right) e_{j}(x) .
\end{gathered}
$$

Here, $\left(e_{j}\right)_{j}$ is an orthonormal basis of $L^{2}(S)$ composed of eigenvectors of $\Delta_{S}$ in $W$ and $\lambda_{j}$ is the eigenvalue associated with $e_{j}$. Unfortunately, this operator is nonlocal and is therefore difficult to handle numerically. A third choice will be presented later.

Remark 2.4. In the simplest case where $\mathscr{S}$ is given by (8) and where $a_{i}$ is associated with a Poisson equation, the transformed problem (5)-(7) is simply

$$
\begin{aligned}
& -\Delta u_{i}=f \text { on } \Omega_{i}, \\
& r\left(u_{i}-q\right)+\lambda_{i}+\frac{\partial u_{i}}{\partial n_{i}}=0 \text { on } S, \\
& q=\frac{1}{2}\left(u_{1}+u_{2}\right)+\frac{1}{2 r}\left(\lambda_{1}+\lambda_{2}\right), \\
& u_{i}=q .
\end{aligned}
$$

Observe in all cases that both subdomains play the same role.

\subsection{Equivalency result. We have}

Theorem 2.1. The variational formulation (1) of the original problem is equivalent to the Lagrangian formulation (4).

Proof. First, let $u \in V_{0}$ be a solution of the original problem (1). We introduce the inverse trace $\operatorname{Tr}_{i}^{-1}: W \rightarrow\left(\operatorname{ker}^{-1} \operatorname{Tr}_{i}\right)^{\perp} \cap V_{i}$. By construction, $\operatorname{Tr}_{i}^{-1}$ is uniquely defined. We now define $\lambda_{i} \in W$ by solving the well-posed problem

$$
\left(\lambda_{i}, \hat{q}\right)=\left\langle\mathscr{S} \lambda_{i}, \hat{q}\right\rangle=a_{j}\left(u_{j}, \operatorname{Tr}_{j}^{-1} \hat{q}\right)-L_{j}\left(\operatorname{Tr}_{j}^{-1} \hat{q}\right) \quad, \quad \forall \hat{q} \in W .
$$

By construction, and since $u$ is a solution of (1), we then have

$$
\left(\lambda_{1}+\lambda_{2}, \hat{q}\right)=\sum_{i=1}^{2} a_{i}\left(u_{i}, \operatorname{Tr}_{i}^{-1} \hat{q}\right)-L_{i}\left(\operatorname{Tr}_{i}^{-1} \hat{q}\right)=0, \forall \hat{q} \in W
$$


On the other hand, setting $q=\operatorname{Tr} u$ on $S$, we have by definition of $\mathscr{L}_{r}$ and $\lambda_{i}$ and from (1)

$$
\begin{aligned}
\frac{\partial \mathscr{L}_{r}}{\partial v_{i}}(u, q, \lambda) \cdot w_{i} & =a_{i}\left(u_{i}, w_{i}\right)+\left\langle\mathscr{S} \lambda_{i}, \operatorname{Tr} w_{i}\right\rangle-L_{i}\left(w_{i}\right) \\
& =a_{i}\left(u_{i}, w_{i}\right)+a_{j}\left(u_{j}, \operatorname{Tr}_{j}^{-1} \operatorname{Tr} w_{i}\right)-L_{j}\left(\operatorname{Tr}_{j}^{-1} \operatorname{Tr} w_{i}\right)-L_{i}\left(w_{i}\right) \\
& =0, \forall w_{i} \in V_{i}
\end{aligned}
$$

By addition, this implies (4) (i). Then, by construction of $\mathscr{L}_{r}$, of $q$, and from (10), we have

$$
\begin{aligned}
\left\langle\frac{\partial \mathscr{L}_{r}}{\partial q}(u, q, \lambda), d q\right\rangle & =-\sum_{i=1}^{2}\left(r\left\langle\mathscr{S}\left(u_{i}-q\right), d q\right\rangle+\left\langle\mathscr{S} \lambda_{i}, d q\right\rangle\right) \\
& =\left\langle\mathscr{S} \lambda_{1}+\mathscr{S} \lambda_{2}, d q\right\rangle=0 \forall d q \in W,
\end{aligned}
$$

which is (4) (ii). Finally, by construction of $q$, we have

$$
\left\langle\frac{\partial \mathscr{L}_{r}}{\partial \lambda}(u, q, \lambda), d \lambda\right\rangle=-\sum_{i=1}^{2}\left\langle\mathscr{S} d \lambda_{i}, u_{i}-q\right\rangle=0 \quad, \quad \forall d \lambda \in H
$$

which is (4) (iii).

Conversely, let $(u, q, \lambda) \in V \times W \times H$ be a solution of (4). From (4) (iii), we first have

$$
\left\langle\mathscr{S} d \lambda_{i}, u_{i}-q\right\rangle=0 \quad, \quad \forall d \lambda_{i}
$$

from which we deduce $\operatorname{Tr} u_{1}=\operatorname{Tr} u_{2}=q$ on the interface $S$. Plugging this back in (4) (ii) then yields

$$
\left\langle\mathscr{S}\left(\lambda_{1}+\lambda_{2}\right), d q\right\rangle=0 \quad, \quad \forall d q \in W .
$$

This relation used with $d q=\operatorname{Tr} w$ combined with (4) (i), and written for $w \in V_{0}$ finally gives

$$
\begin{aligned}
0 & =\frac{\partial \mathscr{L}_{r}}{\partial v}(u, q, \lambda) \cdot w \\
& =\sum_{i} a_{i}\left(u_{i}, w_{i}\right)+r\left\langle\mathscr{S}\left(q-u_{i}\right), w_{i}\right\rangle+\left\langle\mathscr{S} \lambda_{i}, w_{i}\right\rangle-L_{i}\left(w_{i}\right) \\
& =\sum_{i} a_{i}\left(u_{i}, w_{i}\right)-L_{i}\left(w_{i}\right), \forall w \in V_{0}
\end{aligned}
$$

which is (1).

2.4. Solution algorithm. Formulation (4) is particularly interesting because it can be solved by an augmented Lagrangian algorithm with good parallel properties. For example, we can use the following algorithm (called ALG3 in [12] and [16]). 
Algorithm (11)-(14). With $\lambda_{i}^{0}$ and $q^{-1}$ given, then for $n \geq 0$, with $\lambda_{i}^{n}$ and $q^{n-1}$ being given, solve successively

$$
\begin{aligned}
& a_{i}\left(u_{i}^{n}, w_{i}\right)-L_{i}\left(w_{i}\right)+r\left\langle\mathscr{S}\left(u_{i}^{n}-q^{n-1}\right), w_{i}\right\rangle+\left\langle\mathscr{S} \lambda_{i}^{n}, w_{i}\right\rangle=0, \\
& \quad \forall w_{i} \in V_{i}, u_{i}^{n} \in V_{i}, i=1,2, \\
& \lambda_{i}^{n+\frac{1}{2}}=\lambda_{i}^{n}+r\left(u_{i}^{n}-q^{n-1}\right) ; i=1,2, \\
& -r\left\langle\mathscr{S}\left(u_{1}^{n}+u_{2}^{n}-2 q^{n}\right), \hat{q}\right\rangle-\left\langle\mathscr{S}\left(\lambda_{1}^{n+\frac{1}{2}}+\lambda_{2}^{n+\frac{1}{2}}\right), \hat{q}\right\rangle=0, \forall \hat{q}, \\
& \lambda_{i}^{n+1}=\lambda_{i}^{n+\frac{1}{2}}+r\left(u_{i}^{n}-q^{n}\right) ; i=1,2 .
\end{aligned}
$$

For the choice $\rho_{n}=r$, this algorithm has good convergence properties as will be proved later. Its only practical drawback concerns the choice of the operator $\mathscr{S}$ and of the coefficient $r$.

2.5. Equivalence with the fictitious overlapping techniques. By. construction, we can rewrite Step 4 to 2 of algorithm (11)-(14) as follows:

$$
\begin{aligned}
r \mathscr{S} q^{n}-\mathscr{S} \lambda_{i}^{n+1} & =2 r \mathscr{S} q^{n}-\mathscr{S} \lambda_{i}^{n+\frac{1}{2}}-r \mathscr{S} u_{i}^{n} \\
& =r \mathscr{S}\left(u_{j}^{n}+u_{i}^{n}\right)+\mathscr{S}\left(\lambda_{i}^{n+\frac{1}{2}}+\lambda_{j}^{n+\frac{1}{2}}\right)-\mathscr{S} \lambda_{i}^{n+\frac{1}{2}}-r \mathscr{S} u_{i}^{n} \\
& =r \mathscr{S} u_{j}^{n}+\mathscr{S} \lambda_{j}^{n+\frac{1}{2}} \\
& =r \mathscr{S} u_{j}^{n}+\mathscr{S} \lambda_{j}^{n}+r \mathscr{S}\left(u_{j}^{n}-q^{n-1}\right) \\
& =2 r \mathscr{S} u_{j}^{n}+\mathscr{S} \lambda_{j}^{n}-r \mathscr{S} q^{n-1} .
\end{aligned}
$$

On the other hand, integrating the first step of algorithm (11)-(14) by parts, we have

$$
\sigma_{i}^{n} \cdot n_{i}=-r \mathscr{S} u_{i}^{n}+r \mathscr{S} q^{n-1}-\mathscr{S} \lambda_{i}^{n} \quad \text { on } S .
$$

After elimination of $\lambda_{i}$ and $q$, there remains

$$
\begin{aligned}
\sigma_{i}^{n+1} \cdot n_{i}+r \mathscr{S} u_{i}^{n+1} & =r \mathscr{S} q^{n}-\mathscr{S} \lambda_{i}^{n+1} \\
& =r \mathscr{S} u_{j}^{n}+\left(r \mathscr{S} u_{j}^{n}+\mathscr{S} \lambda_{j}^{n}-r \mathscr{S} q^{n-1}\right) \\
& =r \mathscr{S} u_{j}^{n}-\sigma_{j}^{n} \cdot n_{j} .
\end{aligned}
$$

Therefore, Step 1 takes the final form (once integrated by parts)

$$
\begin{aligned}
& \operatorname{div} \sigma\left(\nabla u_{i}^{n+1}\right)+f_{i}=0 \quad \text { on } \Omega_{i}, \\
& u_{i}^{n+1}=0 \quad \text { on } \partial \Omega_{D_{i}}, \\
& \sigma_{i}^{n+1} \cdot n_{i}=g \quad \text { on } \partial \Omega_{N} \cap \partial \Omega_{i}, \\
& \sigma_{i}^{n+1} \cdot n_{i}+r \mathscr{S} u_{i}^{n+1}=r \mathscr{S} u_{j}^{n}-\sigma_{j}^{n} \cdot n_{j} \quad \text { on } S .
\end{aligned}
$$

This is precisely the nonoverlapping Schwarz alternating method proposed by P.L. Lions [20] (with $\lambda_{i j}=r \mathscr{S}$ ). Therefore, as observed in [16], algorithm (11)-(14) and the nonoverlapping Schwarz alternating method correspond to the same algorithm. 
Remark 2.5. An alternative algorithm for solving (4) consists in eliminating $u_{i}$. The resulting dual problem in $\lambda$ can then be solved by the FETI method of Farhat and Roux [11].

\section{CONVERgENCE RESUlts}

The solution of the original problem (1) by Algorithm (11)-(14) can now be interpreted either as the numerical integration of the associated dual problem by alternating direction methods or as the nonoverlapping Schwarz alternating method proposed by P.L. Lions [20] and studied in [15]. The first analogy appears to be very useful both from theoretical and practical points of view, since it leads to stronger convergence results in the case where $\partial \Omega_{D_{i}}$ is nonempty.

\subsection{Equivalence between augmented Lagrangian and alternating direction meth-} ods for the dual problem. As seen in Glowinski and Le Tallec [16], alternating direction methods are difficult to write in a general augmented Lagrangian setting if $(B \neq$ Id). Following Gabay [13], we shall overcome this difficulty by considering a dual formulation. For that purpose, let us formalize our notation and assumption.

Notation. We introduce the space $E$, the function $\mathscr{F}$ and the operators $B$, $A_{1}$ and $A_{2}$ as follows:

$$
E=\left\{q=\left(q_{12}, q_{21}\right) \in H, q_{12}=q_{21}\right\},
$$

$\mathscr{F}=\operatorname{Ind}_{E}=$ Indicator function of $E$ in $H$, with subgradient $\partial \mathscr{F}=A_{1}^{-1}$,

$$
\begin{gathered}
B: V=V_{1} \times V_{2} \rightarrow H=W^{2}, \\
w=\left(w_{1}, w_{2}\right) \rightarrow\left(\operatorname{Tr} w_{1}, \operatorname{Tr} w_{2}\right), \\
A_{2}: H \rightarrow H, \\
\lambda \rightarrow-B u(\lambda),
\end{gathered}
$$

with $u(\lambda)$ a solution of the domain decomposed elliptic problem

$$
\sum_{i}\left(a_{i}\left(u(\lambda), w_{i}\right)-L_{i}\left(w_{i}\right)\right)+(\lambda, B w)=0 \quad, \quad \forall w \in V, u(\lambda) \in V .
$$

We will assume (Assumption 3.1) that the bilinear form $\sum_{i} a_{i}(v, w)$ is coercive and continuous on the product space $V=V_{1} \times V_{2}$. For most operators, and in particular for elasticity problems, this brings some restriction on the choice of the splitting $\Omega=\Omega_{1} \cup \Omega_{2}$. Mainly, each domain $\Omega_{i}$ must be fixed on part of its boundary. Now, we are ready to use the general results of Gabay or of Glowinski and Le Tallec, which take here the form of

Theorem 3.1. The Lagrangian formulation (4) is equivalent to the dual problem

$$
0 \in A_{1}(\lambda)+A_{2}(\lambda) \text { in } H .
$$

Proof. We have observed in Theorem 2.1 that the solution of (4) is independent of $r$, hence we can take $r=0$ in (4). Then from (4) (iii), we have $\operatorname{Tr} u_{1}=$ 
$\operatorname{Tr} u_{2}=q$ and hence

$$
B u=\left(\operatorname{Tr} u_{1}, \operatorname{Tr} u_{2}\right) \in E .
$$

Now writing (4)(i) with $r=0$, we get by definition of $\mathscr{L}_{0}$

$$
\sum_{i}\left(a_{i}\left(u, w_{i}\right)-L_{i}\left(w_{i}\right)\right)+(\lambda, B w)=0 \quad, \quad \forall w \in V,
$$

or equivalently

$$
-B u=A_{2}(\lambda) \text {. }
$$

On the other hand, writing (4) (ii) with $r=0$ yields

$$
\lambda_{1}+\lambda_{2}=0
$$

and thus $\lambda$ belongs to $E^{\perp}$. Since $B u \in E$, this implies by definition of $\mathscr{F}$ and of its subgradient that

$$
\lambda \in \partial \mathscr{F}(B u)
$$

or equivalently

$$
B u \in A_{1}(\lambda) \text {. }
$$

After elimination of $u$ between the two inclusions, there results

$$
-A_{2}(\lambda) \in A_{1}(\lambda) \text {, }
$$

in which we recognize (15).

Conversely, let $\lambda$ be solution of (15). We first get (4) (i) by setting $u=u(\lambda)$. If we then plug the definition of $u$ in (15), we get

$$
B u \in \partial \mathscr{F}^{-1}(\lambda) \text {, }
$$

which implies $\lambda \in \partial \mathscr{F}(B u)$, that is,

$$
\begin{array}{ll}
\lambda \in E^{\perp} & (\Leftrightarrow(4)(\mathrm{ii})), \\
B u \in E & (\Leftrightarrow(4) \text { (iii)). }
\end{array}
$$

Thus, $(u, \operatorname{Tr} u, \lambda)$ is solution of $(4)$.

\subsection{Linear convergence of algorithm (11)-(14).}

Theorem 3.2. Algorithm (11)-(14) of $\S 2.4$ is equivalent to the multiplicative algorithm

$$
\lambda^{n+1}=\left(I+r A_{1}\right)^{-1}\left(I-r A_{2}\right)\left(I+r A_{2}\right)^{-1}\left(I-r A_{1}\right) \lambda^{n} .
$$

Proof. We follow the steps of the general theory. By construction, algorithm (11)-(14) has the form

$$
\begin{gathered}
\sum_{i}\left(a_{i}\left(u^{n}, w_{i}\right)-L\left(w_{i}\right)\right)+\left\langle r\left(B u^{n}-q^{n-1}\right)+\lambda^{n}, B w\right\rangle=0, \quad \forall w \in V \\
\lambda^{n+\frac{1}{2}}=\lambda^{n}+r\left(B u^{n}-q^{n-1}\right), \\
\partial \mathscr{F}\left(q^{n}\right) \ni r\left(B u^{n}-q^{n}\right)+\lambda^{n+\frac{1}{2}} \\
\lambda^{n+1}=\lambda^{n+\frac{1}{2}}+r\left(B u^{n}-q^{n}\right) .
\end{gathered}
$$


For (19), we recall that the identity $\lambda \in \partial \mathscr{F}(q)$ is equivalent to the identities

$$
q \in E \text { and } \lambda_{1}+\lambda_{2}=0 \text {. }
$$

Replacing now $r\left(B u^{n}-q^{n-1}\right)$ by $\lambda^{n+\frac{1}{2}}-\lambda^{n}$ in (17), and $r\left(B u^{n}-q^{n}\right)$ by $\lambda^{n+1}-$ $\lambda^{n+\frac{1}{2}}$ in (19), we get

$$
\begin{gathered}
u^{n}=u\left(\lambda^{n+\frac{1}{2}}\right), \\
\lambda^{n+1} \in \partial \mathscr{F}\left(q^{n}\right) \Leftrightarrow q^{n} \in A_{1}\left(\lambda^{n+1}\right),
\end{gathered}
$$

respectively. In view of (21)-(22), the relations (18) and (20) now become

$$
\begin{gathered}
\frac{\left(\lambda^{n+\frac{1}{2}}-\lambda^{n}\right)}{r}+A_{2}\left(\lambda^{n+\frac{1}{2}}\right)+A_{1}\left(\lambda^{n}\right) \ni 0, \\
\frac{\left(\lambda^{n+1}-\lambda^{n+\frac{1}{2}}\right)}{r}+A_{2}\left(\lambda^{n+\frac{1}{2}}\right)+A_{1}\left(\lambda^{n+1}\right) \ni 0 .
\end{gathered}
$$

Eliminating $\lambda^{n+\frac{1}{2}}$ then leads to (16).

This is the form introduced in [21], and on which our convergence analysis will be based.

Now, we are ready to prove the main result of this section, that is the linear convergence of algorithm (11)-(14), when written in the form (16).

Theorem 3.3. Under Assumption 3.1 and if $(\cdot, \cdot)$ is equivalent to the $H^{1 / 2}(S)$ scalar product, $A_{2}$ is coercive and Lipschitz continuous on $H$, with constants $\alpha$ and $C$. Moreover, the sequence $\left(\lambda^{n}\right)$ defined by (16) converges strongly to a solution $\lambda$ of the dual problem (15), and we have

$$
\left|\lambda^{n}-\lambda\right|_{H} \leq C_{0}\left(1-\frac{4 r \alpha}{(1+C r)^{2}}\right)^{\frac{n}{2}}\left|\lambda^{0}-\lambda\right|_{H} .
$$

There also exists an optimal parameter $r^{*}$ for which we have

$$
\left|\lambda^{n}-\lambda\right|_{H} \leq C_{0}\left(1-\frac{\alpha}{C}\right)^{\frac{n}{2}}\left|\lambda^{0}-\lambda\right|_{H} .
$$

Proof. Step 1. By definition of $A_{2}$, we first get

$$
\left|A_{2}(\lambda)-A_{2}(\tilde{\lambda})\right|_{H}=|B u-B \tilde{u}|_{H} \leq\|B\|\|u-\tilde{u}\|_{V} .
$$

On the other hand, from Assumption 3.1, we have

$$
\begin{aligned}
\left(A_{2}(\lambda)-A_{2}(\tilde{\lambda}), \lambda-\tilde{\lambda}\right) & =-(B(u-\tilde{u}), \lambda-\tilde{\lambda}) \\
& =-B^{t}(\lambda-\tilde{\lambda}) \cdot(u-\tilde{u}) \\
& =\sum_{i} a_{i}(u-\tilde{u}, u-\tilde{u}) \\
& \geq \alpha_{0}\|u-\tilde{u}\|_{V}^{2} .
\end{aligned}
$$


Using now (26) combined with (25) yields

$$
\begin{aligned}
\alpha_{o}\|u-\tilde{u}\|_{V}^{2} & \leq\left(A_{2}(\lambda)-A_{2}(\tilde{\lambda}), \lambda-\tilde{\lambda}\right) \\
& \leq\left|A_{2}(\lambda)-A_{2}(\tilde{\lambda})\right||\lambda-\tilde{\lambda}| \\
& \leq\|B\|\|u-\tilde{u}\|_{V}|\lambda-\tilde{\lambda}|
\end{aligned}
$$

from which we deduce

$$
\begin{aligned}
\|u-\tilde{u}\|_{V} & \leq \frac{\|B\|}{\alpha_{o}}|\lambda-\tilde{\lambda}| \\
\left|A_{2} \lambda-A_{2} \tilde{\lambda}\right| & \leq \frac{\|B\|^{2}}{\alpha_{o}}|\lambda-\tilde{\lambda}| .
\end{aligned}
$$

This is the desired Lipschitz continuity with constant $C=\|B\|^{2} / \alpha_{0}$.

To check the coercivity, we introduce

$$
v-\tilde{v}=B^{-1}(\lambda-\tilde{\lambda}) .
$$

By construction of $u$, we then have

$$
\begin{aligned}
|\lambda-\tilde{\lambda}|^{2} & =\left(B B^{-1}(\lambda-\tilde{\lambda}), \lambda-\tilde{\lambda}\right) \\
& =\left(v-\tilde{v}, B^{t}(\lambda-\tilde{\lambda})\right) \\
& =-\sum_{i} a_{i}(u-\tilde{u}, v-\tilde{v}) \\
& \leq\|v-\tilde{v}\|_{V} M\|u-\tilde{u}\|_{V}, \\
& \leq\left\|B^{-1}\right\| M|\lambda-\tilde{\lambda}|\|\tilde{u}-u\|_{V} .
\end{aligned}
$$

Here, $M$ denotes the constant of continuity of $\sum_{i} a_{i}(.,$.$) . From this, we get$

$$
|\lambda-\tilde{\lambda}|_{H} \leq M\left\|B^{-1}\right\|\|u-\tilde{u}\|_{V}
$$

which, plugged back in (26), yields

$$
\left(A_{2}(\lambda)-A_{2}(\tilde{\lambda}), \lambda-\tilde{\lambda}\right) \geq \alpha_{0}\left\|B^{-1}\right\|^{-2} M^{-2}|\lambda-\tilde{\lambda}|_{H}^{2} .
$$

Hence the coercivity of $A_{2}$ with constant $\alpha=\alpha_{0}\left\|B^{-1}\right\|^{-2} M^{-2}$. The above proof uses the continuity of $B$ and $B^{-1}$, which is a direct consequence of the trace theorem as soon as $H$ is endowed with a $H^{\frac{1}{2}}(S)$ equivalent scalar product.

Step 2: Convergence. Following [21], we introduce

$$
\begin{array}{rlrl}
a_{2} & =A_{2}(\lambda), & a_{2}^{n} & =A_{2}\left(\lambda^{n}\right), \\
a_{1} & =-a_{2}, & & \\
\beta & =\lambda+r a_{2}, & \beta^{n} & =r a_{2}^{n}+\lambda^{n}, \\
\alpha & =\lambda+r a_{1}, & \alpha^{n}=2 \lambda^{n}-\beta^{n}, \\
a_{1}^{n} & =\left(\alpha^{n}-\beta^{n+1}\right) / 2 r . & &
\end{array}
$$

Since (1) has a solution, we know by equivalence that (15) has a solution $\lambda$, and hence the above quantities are well defined. 
Using Proposition 1 of [21], we have

$$
\begin{gathered}
0 \leq\left(a_{2}^{n}-a_{2}, \lambda^{n}-\lambda\right)=\frac{1}{4 r}\left(\left|\beta^{n}-\beta\right|^{2}-\left|\alpha^{n}-\alpha\right|^{2}\right), \\
0 \leq\left(a_{1}^{n}-a_{1}, \frac{\beta^{n+1}+\alpha^{n}}{2}-\lambda\right)=\frac{1}{4 r}\left(\left|\alpha^{n}-\alpha\right|^{2}-\left|\beta^{n+1}-\beta\right|^{2}\right) .
\end{gathered}
$$

By addition, and from the coercivity of $A_{2}$, we deduce

$$
\alpha\left|\lambda^{n}-\lambda\right|^{2} \leq\left(a_{2}^{n}-a_{2}, \lambda^{n}-\lambda\right) \leq \frac{1}{4 r}\left(\left|\beta^{n}-\beta\right|^{2}-\left|\beta^{n+1}-\beta\right|^{2}\right) .
$$

On the other hand, from the Lipschitz continuity of $A_{2}$, we obtain

$$
\left|\beta^{n}-\beta\right|=\left|\left(\lambda^{n}-\lambda\right)+r\left(a_{2}^{n}-a_{2}\right)\right| \leq(1+r C)\left|\lambda^{n}-\lambda\right| .
$$

Plugging this in (28) yields finally

$$
\frac{1}{4 r}\left(\left|\beta^{n}-\beta\right|^{2}-\left|\beta^{n+1}-\beta\right|^{2}\right) \geq(1+r C)^{-2} \alpha\left|\beta^{n}-\beta\right|^{2},
$$

that is,

$$
\left|\beta^{n+1}-\beta\right|^{2} \leq\left(1-\frac{4 r \alpha}{(1+r C)^{2}}\right)\left|\beta^{n}-\beta\right|^{2} .
$$

For any value of $r$, we thus have that $\beta^{n}$ is a converging sequence, which converges at least linearly with constant

$$
\left(1-\frac{4 r \alpha}{(1+r C)^{2}}\right)^{\frac{1}{2}}
$$

This constant attains the minimum value $(1-\alpha / C)$ for the choice $r^{*}=1 / C$.

To deduce linear convergence of $\lambda^{n}$, one simply writes

$$
\begin{aligned}
\left|\lambda^{n}-\lambda\right|^{2}(1+r \alpha) & \leq\left|\lambda^{n}-\lambda\right|^{2}+r\left(a_{2}^{n}-a_{2}, \lambda^{n}-\lambda\right) \\
& \leq\left(\beta^{n}-\beta, \lambda^{n}-\lambda\right) \\
& \leq\left|\beta^{n}-\beta \| \lambda^{n}-\lambda\right|,
\end{aligned}
$$

which yields

$$
\left|\lambda^{n}-\lambda\right| \leq(1+r \alpha)^{-1}\left|\beta^{n}-\beta\right|,
$$

and hence (23) (from (29)).

Remark 3.1. The above constants depend on the subdomain diameters and aspect ratios through the norms $\|B\|$ and $\left\|B^{-1}\right\|$. Hence, they do not scale well when the number of subdomains increases. The present version of algorithm (11)-(14) is therefore not adaptable to the many-subdomains case.

\section{LAGRANGIAN APPROACH OF THE DISCRETE PROBLEM}

4.1. The standard conforming case. To approximate the variational problem (1) by finite element methods, the standard procedure introduces a global finite 

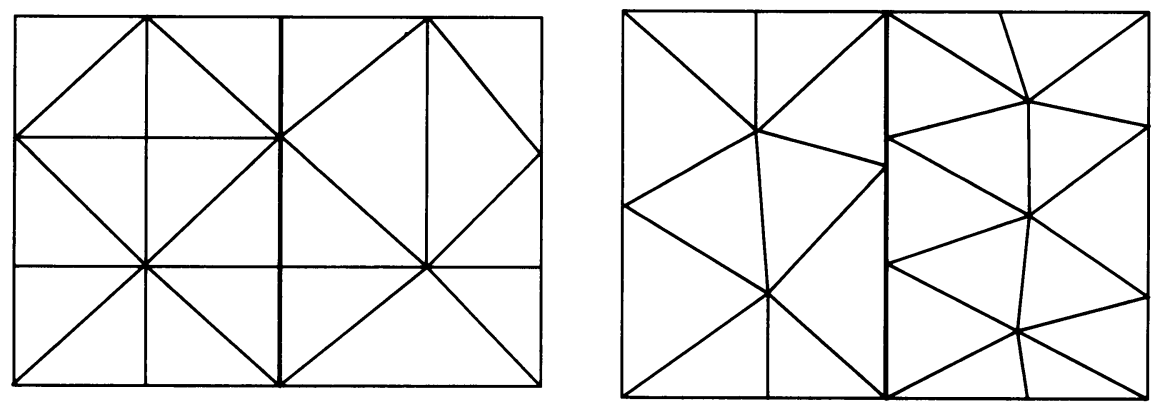

FIGURE 2. Matching and nonmatching grids

element approximation $V_{0 h}$ of $V_{0}$. If the subdomain interfaces coincide with grid lines (Figure 2: matching grids), it is then easy to define restriction spaces

$$
V_{i h}=\left\{v_{i h}=v_{0 h \mid \Omega_{i}}, v_{0 h} \in V_{0 h}\right\},
$$

the product space

$$
V_{h}=V_{1 h} \times V_{2 h},
$$

and the trace space

$$
W_{h}=\operatorname{Tr} V_{1 h}\left|S=\operatorname{Tr} V_{2 h}\right| S .
$$

We then have as in the continuous case

$$
V_{0 h}=\left\{\left(v_{1 h}, v_{2 h}\right) \in V_{1 h} \times V_{2 h}, \operatorname{Tr} v_{1 h \mid S}=\operatorname{Tr} v_{2 h \mid S}\right\}
$$

and problem (1) is approximated by

$$
\sum_{i}\left\{a_{i}\left(u_{h}, v_{h}\right)-L_{i}\left(v_{h}\right)\right\}=0 \quad, \quad \forall v_{h} \in V_{0 h}, u_{h} \in V_{0 h} .
$$

4.2. The discrete problem for nonmatching grids. To approximate (1), we can also replace $V_{i}$ by independent (nonmatching) Conforming Element Spaces $V_{i h}$, introduce a discrete auxiliary space $W_{h}$ defined on the interface $S$ (close to the so-called mortar elements [1]) and replace in the continuous problem (1) the space $V_{0}$ by its approximation $V_{0 h}$ given by

$$
V_{0 h}=\left\{\left(v_{1 h}, v_{2 h}\right) \in V_{1 h} \times V_{2 h}, \int_{S}\left(\operatorname{Tr} v_{1 h}-\operatorname{Tr} v_{2 h}\right) w_{h}=0, \forall w_{h} \in W_{h}\right\} .
$$

The discrete problem is then

$$
\sum_{i}\left\{a_{i}\left(u_{h}, v_{h}\right)-L_{i}\left(v_{h}\right)\right\}=0, \quad \forall v_{h} \in V_{0 h}, u_{h} \in V_{0 h} .
$$

We still obtain the classical equilibrium equation in $V_{0 h}$, but this space is not classical and is not included in $V_{0}$. In other words, the continuity of the discrete solution and of the test functions at the interfaces is imposed in a weak sense only. Such an approximation may or may not be included in $V_{0}$, depending on the choice of the interface space $W_{h}$ (for more details see [1] or [18]). The approximate problem (30) has already been introduced and studied in $\S 2$ of [18] or in [19], where it was proved that (30) defines a consistent nonconforming approximation of (1) . 
For the following, in order to get a common notation for matching and nonmatching grids, it will be convenient to introduce the discrete trace operator $\operatorname{Tr}_{i h}$ defined from $V_{i h}$ into $W_{h}$ and which to a given $v_{i h} \in V_{i h}$ associates its $L^{2}$ projection $\operatorname{Tr}_{i h} v_{i h}$ onto $W_{h}$. With this new notation, the space $V_{0 h}$ is then defined in both cases as

$$
V_{0 h}=\left\{\left(v_{1 h}, v_{2 h}\right) \in V_{1 h} \times V_{2 h}, \operatorname{Tr}_{1 h} v_{1 h}=\operatorname{Tr}_{2 h} v_{2 h}\right\} .
$$

4.3. Lagrangian formulation. The augmented Lagrangian approach of $\S 2$ is able to treat matching and nonmatching grids in the same framework. For this purpose, it replaces $V, W$ and $H$ by the above finite-dimensional subspaces $V_{h}=V_{1 h} \times V_{2 h}, W_{h}$ and $H_{h}=W_{h}^{2}$. Then, the discrete Lagrangian formulation of (30) is:

Find $\left(u_{h}, q_{h}, \lambda_{h}\right) \in V_{h} \times W_{h} \times H_{h}$ such that

$$
\left\{\begin{array}{rll}
\text { (i) } \frac{\partial \mathscr{L}_{r}^{h}}{\partial v}\left(u_{h}, q_{h}, \lambda_{h}\right) \cdot w_{h}=0, & \forall w_{h} \in V_{h}, \\
\text { (ii) }\left\langle\frac{\partial \mathscr{L}_{r}^{h}}{\partial q}\left(u_{h}, q_{h}, \lambda_{h}\right), d q_{h}\right\rangle=0, & \forall d q_{h} \in W_{h}, \\
\text { (iii) }\left\langle\frac{\partial \mathscr{L}_{r}^{h}}{\partial \lambda}\left(u_{h}, q_{h}, \lambda_{h}\right), d \lambda_{h}\right\rangle=0, & \forall d \lambda_{h} \in H_{h},
\end{array}\right.
$$

and can again be solved by Algorithm (11)-(14). Here, the augmented Lagrangian $\mathscr{L}_{r}^{h}$ is defined by

$$
\begin{aligned}
\mathscr{L}_{r}^{h}\left(v_{i h}, q_{i h}, \lambda_{i h}\right)=\sum_{i}\left\{\frac{1}{2} a_{i}\left(v_{i h}, v_{i h}\right)-L_{i}\left(v_{i h}\right)+\right. & \frac{r}{2}\left|\operatorname{Tr}_{i h} v_{i h}-q_{h}\right|_{h}^{2} \\
& \left.+\left(\lambda_{i h}, \operatorname{Tr}_{i h} v_{i h}-q_{h}\right)_{h}\right\} .
\end{aligned}
$$

The space $W_{h}$ is endowed with the scalar product

$$
\left(q_{h}, \hat{q}_{h}\right)_{h}=\left\langle\mathscr{S}_{h} q_{h}, \hat{q}_{h}\right\rangle,
$$

with $\mathscr{S}_{h}$ a positive selfadjoint operator defined from $W_{h}$ into $W_{h}^{\prime}$ and to be specified later.

Remark 4.1. The choices of $r$ and $\mathscr{S}_{h}$ play no role from the theoretical point of view, but will be critical to ensure good convergence of Algorithm (11)-(14).

For the time being we make the assumptions:

Assumption 4.1. The scalar product $(\cdot, \cdot)_{h}$ is equivalent to the $H^{1 / 2}(S)$ scalar product, uniformly in $h$, that is, there exist constants $C_{1}$ and $C_{2}$ independent of $h$ such that

$$
C_{1}\left\|q_{h}\right\|_{W}^{2} \leq\left\langle\mathscr{S}_{h} q_{h}, q_{h}\right\rangle \leq C_{2}\left\|q_{h}\right\|_{W}^{2} \quad, \quad \forall q_{h} \in W_{h} .
$$

Assumption 4.2. The functions of $W_{h}$ take zero values on $\partial \Omega_{D}$, and there exist strictly positive constants $\beta_{i}$ independent of $h$ such that

$$
\inf _{q_{h} \in W_{h}} \sup _{v_{i h} \in V_{i h}}\left\{\frac{\int_{S} q_{h} v_{i h} d a}{\left\|q_{h}\right\|_{L^{2}(S)}\left\|v_{i h}\right\|_{L^{2}(S)}}\right\} \geq \beta_{i} .
$$


The first assumption requires us again to choose a discrete scalar product which behaves like the $H^{1 / 2}(S)$ product. The second has already been encountered in the numerical analysis of (30) and requires that the interface space $W_{h}$ not be too large with respect to the spaces $\operatorname{Tr} V_{i h}$. More precisely, this assumption means that the discrete trace $\operatorname{Tr}_{i h}$ is still a continuous surjection from $V_{i h}$ onto $W_{h}$. It is automatically satisfied in the conforming case.

Assumption 4.3. The finite element space $W_{h}$ appearing in Assumption 4.2 is constructed on a uniformly regular triangulation. In other words, in twodimensional geometries, there exists a constant $C>0$ such that, for any triangle $K$ in $\mathscr{T}_{h}$ for which $K \cap \bar{S}$ is a whole edge of $K$, we have

$$
l(K \cap \bar{S}) \geq C h .
$$

Here, $l(K \cap \bar{S})$ denotes the length of the segment $K \cap \bar{S}$.

With these assumptions, and assuming that $V_{i h}$ is a regular finite element space in the sense of [2], we have the following preliminary lemmas, proved in the Appendix:

Lemma 4.1. With the above assumptions, we have

$$
\left\|\operatorname{Tr}_{i h} v_{h}\right\|_{W} \leq C_{3}\left\|v_{h}\right\|_{V_{i}}, \forall v_{h} \in V_{i h},
$$

with $C_{3}$ independent of $h$.

Lemma 4.2. Under Assumptions 4.1, 4.2 and 4.3, the trace operator $\operatorname{Tr}_{i h}$ has an inverse $\operatorname{Tr}_{i h}^{-1}$ which satisfies

$$
\left\|\operatorname{Tr}_{i h}^{-1} w_{h}\right\|_{V_{i}} \leq C\left\|w_{h}\right\|_{W}, \forall w_{h} \in W_{h},
$$

with $C$ independent of $h$.

\subsection{Uniform linear convergence of the discrete algorithm.}

Theorem 4.1. Under Assumptions 3.1, 4.1, 4.2 and 4.3, Algorithm (11)-(14) applied to (31) converges linearly uniformly in $h$, that is,

$$
\left|\lambda^{n+1}-\lambda\right|_{h} \leq\left(1-\frac{4 r \bar{\alpha}}{(1+\bar{C} r)^{2}}\right)^{1 / 2}\left|\lambda^{n}-\lambda\right|_{h} .
$$

Here the constants $\bar{\alpha}$ and $\bar{C}$ do not depend on $h$.

Proof. The proof is the same as in the continuous case when $V_{i}$ is replaced by $V_{i h}$ and $\operatorname{Tr}_{i}$ by $\operatorname{Tr}_{i h}$. Hence, we first obtain the linear convergence of the algorithm with constants

$$
\begin{aligned}
\alpha_{h} & =\frac{\gamma}{M^{2}\left\|B_{h}^{-1}\right\|_{h}^{2}}, \\
C_{h} & =\frac{\left\|B_{h}\right\|_{h}^{2}}{\gamma},
\end{aligned}
$$

under the notation

$$
\left\|B_{h}\right\|_{h}=\sup _{v_{h} \in V_{h}} \frac{\left\|B_{h} v_{h}\right\|_{h}}{\left\|v_{h}\right\|_{1, \Omega}}
$$




$$
\left\|B_{h}^{-1}\right\|_{h}=\sup _{w_{h} \in W_{h}} \frac{\left\|B_{h}^{-1} w_{h}\right\|_{1, \Omega}}{\left\|w_{h}\right\|_{h}} .
$$

But here, we have

$$
B_{h} v_{h}=\left(\operatorname{Tr}_{1 h} v_{1 h}, \operatorname{Tr}_{2 h} v_{2 h}\right)
$$

Hence, we indeed have $\left\|B_{h}\right\|_{h}$ bounded independently of $h$ as a consequence of Lemma 4.1 and Assumption 4.1. Similarly, the boundedness of $\left\|B_{h}^{-1} w_{h}\right\|_{h}$ independently of $h$ is a direct consequence of Lemma 4.2. Therefore, the constant $\alpha_{h}$ (resp. $C_{h}$ ) is bounded below (resp. above) by $\bar{\alpha}$ (resp. $\bar{C}$ ) independently of $h$, and our theorem is proved.

4.5. A simplified choice of $\mathscr{S}_{h}$. The operator $\mathscr{S}_{h}$ acts numerically in Algorithm (11)-(14) through the combination $\operatorname{Tr}_{i}^{T} \mathscr{S}_{h} \operatorname{Tr}_{j}$. Therefore, our first idea is to ignore the equivalence condition stated in Assumption 4.1 and to choose $\mathscr{S}_{h}$ in order to get the simplest possible operator $\operatorname{Tr}_{i}^{T} \mathscr{S}_{h} \operatorname{Tr}_{j}$.

To this end, using the nodal basis $\left(\psi_{i}^{p}\right)_{p}$ and $\left(\phi^{l}\right)_{l}$ of $V_{i h}$ and $W_{h}$, we first define the matrices

$$
\begin{aligned}
B_{i}^{l q} & =\int_{S} \phi^{l} \psi_{i}^{q} d x, \\
M_{W}^{m l} & =\int_{S} \phi^{l} \phi^{m} d x .
\end{aligned}
$$

These "mass" matrices define the $L^{2}$ scalar product on $\operatorname{Tr} V_{i h} \times W_{h}$ and $W_{h} \times$ $W_{h}$, respectively. With these matrices, the $L^{2}$ projection $\operatorname{Tr}_{i h} v_{i h}=\operatorname{Tr}_{i} V_{i}$ of an element $v_{i h}$ of $V_{i h}$ is characterized by

$$
M_{W} \operatorname{Tr}_{i} V_{i}=B_{i} V_{i}
$$

which means that we have

$$
\operatorname{Tr}_{i}=M_{W}^{-1} B_{i}
$$

Now a very simple explicit choice of $\operatorname{Tr}_{i}^{T} \mathscr{S}_{h} \operatorname{Tr}_{j}$ is certainly to take

$$
\operatorname{Tr}_{i}^{T} \mathscr{S}_{h} \operatorname{Tr}_{j}=B_{i}^{T} B_{j}
$$

corresponding to the matrix

$$
\mathscr{S}_{h}=M_{W}^{2} \text {. }
$$

This construction depends on the choice of the nodal basis, has no equivalence in terms of operators, and therefore cannot satisfy Assumption 4.1. But it is very simple, and this is the reason why we have tested it in our numerical tests.

To study the convergence of Algorithm (11)-(14) in this case, we have to estimate $\left\|B_{h}\right\|_{h}$ and $\left\|B_{h}^{-1}\right\|_{h}$. By construction, we first have for all $w_{h}$ in $W_{h}$

$$
\begin{gathered}
\left\|w_{h}\right\|_{h}^{2}=\left\langle M_{W}^{2} W, W\right\rangle, \\
\left\|w_{h}^{2}\right\|_{L^{2}(S)}^{2}=\left\langle M_{W} W, W\right\rangle,
\end{gathered}
$$

with $W$ the vector of nodal values of $w_{h}$. Since the mass matrix $M_{W}$ always has its eigenvalues in the segment $\left[c h^{\mathrm{dim}-1}, C h^{\mathrm{dim}-1}\right]$ with $c$ and $C$ 
independent of $h$, we deduce

$$
c h^{\operatorname{dim}-1}\|w\|_{L^{2}(S)}^{2} \leq\left\|w_{h}\right\|_{h}^{2} \leq C h^{\operatorname{dim}-1}\|w\|_{L^{2}(S)}^{2} .
$$

Then, we first have

$$
\begin{aligned}
\left\|B_{h}\right\|_{h}^{2} & =\sup _{v \in V_{h}} \frac{\left\|B_{h} v\right\|_{h}^{2}}{\|v\|_{H^{1}}^{2}} \\
& \leq C h^{\text {dim-1 }} \sup _{v \in V_{h}} \frac{\left\|B_{h} v\right\|_{L^{2}(S)}^{2}}{\|v\|_{H^{1}}^{2}} \\
& \leq C h^{\operatorname{dim}-1} \sup _{v \in V_{h}} \frac{\|\operatorname{Tr} v\|_{L^{2}(S)}^{2}}{\|v\|_{H^{1}}^{2}}=\mathscr{O}\left(h^{\operatorname{dim}-1}\right) .
\end{aligned}
$$

On the other hand, from Assumption 4.2 and the inverse Sobolev inequality on $W_{h}$, we have

$$
\begin{aligned}
\left\|B_{h}^{-1}\right\|_{h}^{2} & =\sup _{v \in \operatorname{Im} B_{h}^{-1}} \frac{\|v\|_{H^{1}}^{2}}{\left\|B_{h} v\right\|_{h}^{2}} \\
& \leq \frac{C}{c h^{\text {dim }-1}} \sup _{v_{h}} \frac{\|\operatorname{Tr} v\|_{H^{1 / 2}}^{2}}{\left\|B_{h} v\right\|_{L^{2}(S)}^{2}} \\
& \leq \frac{C}{c h^{\text {dim }}} \sup _{v_{h}} \frac{\|\operatorname{Tr} v\|_{L^{2}}^{2}}{\left\|B_{h} v\right\|_{L^{2}(S)}^{2}}=\mathscr{O}\left(\frac{1}{h^{\text {dim }}}\right) .
\end{aligned}
$$

Hence, the linear convergence of Algorithm (11)-(14) is obtained with constants

$$
r=\frac{1}{C_{h}} \approx \mathscr{O}\left(\frac{1}{h^{\operatorname{dim}-1}}\right)
$$

and

$$
\begin{aligned}
C^{\prime}=\left(1-\frac{\alpha_{h}}{C_{h}}\right) & =\left(1-\frac{\left\|B_{h}^{-1}\right\|_{h}^{-2} \gamma^{2}}{\left\|B_{h}\right\|_{h}^{2} M^{2}}\right) \\
& \approx\left(1-\frac{h^{\operatorname{dim}} C}{h^{\operatorname{dim}-1}}\right) \\
& \approx(1-h) .
\end{aligned}
$$

Remark 4.2. The choice $\mathscr{S}_{h}=$ Id will lead to a matrix $\operatorname{Tr}_{i}^{T} \mathscr{S}_{h} \operatorname{Tr}_{j}=$ $B_{i}^{T} M_{W}^{-1} B_{j}$ and is not practical. We could replace $M_{W}$ by a diagonal lumped mass matrix but then we recover our previous choice within the factor $\left(\frac{1}{h}\right)^{\operatorname{dim}-1}$.

\section{INTRODUCTION OF A FICTITIOUS SCHUR PROBLEM}

5.1. The fictitious Steklov-Poincaré operator. It is worth discussing the effective choice of the operator $\mathscr{S}_{h}$ : let us first indicate that this is by and large an open problem. In our case, some examples may be eliminated :

- the choice $\mathscr{S}_{h}=\left(-\Delta_{S}^{h}\right)^{1 / 2}$, where $-\Delta_{S}^{h}$ stands for the discrete LaplaceBeltrami operator on $S$, is theoretically correct but is very impractical in $3 \mathrm{D}$ situations; 


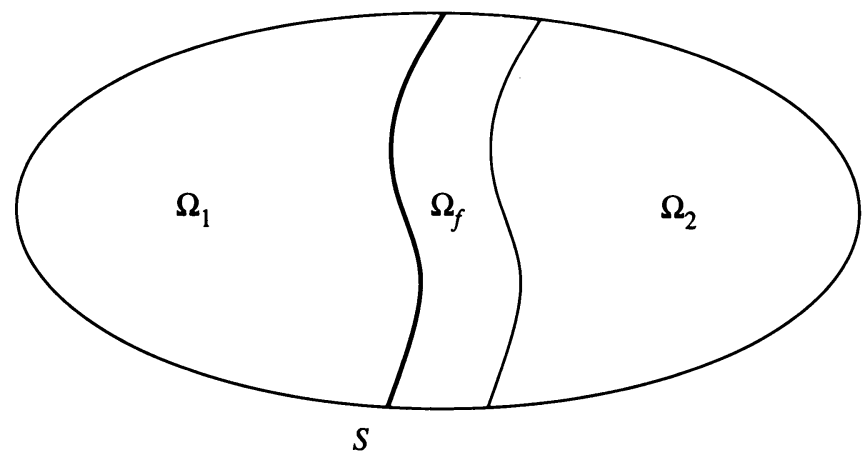

FIGURE 3

- the choice $\mathscr{S}_{h}=\sum_{i=1}^{2} S_{i h}$, where $S_{i h}$ is the discrete Steklov-Poincare operator introduced in [3], is not local and is defined implicitly. This makes the problem in displacement difficult (we must invert the elasticity operator for any degree of freedom located on the interface $S$ ).

The above Steklov-Poincare operator is not practical if the domains $\Omega_{i}$ are too large, but it has interesting features. Mainly, it can be defined for any geometry and for any elliptic operator, including three-dimensional anisotropic heterogeneous elasticity, and it is a coercive positive selfadjoint operator defined on the interface space $W_{h}$.

But then, for each face $S$, we can create in the spirit of Nepomnyaschikh [22] an artificial "dream" domain $\Omega_{f}$ on which to define this Steklov-Poincaré operator. Therefore, with each face $S$, we associate a fictitious three-dimensional domain $\Omega_{f}$ having $S$ as one of its faces. This domain is to be endowed with a finite element space $H_{h}^{1}\left(\Omega_{f}\right)$ and with an elasticity tensor $A_{f}$.

Notation. The domain $\Omega$ is decomposed as indicated in Figure 3, the fictitious domain being denoted by $\Omega_{f}$.

We now define the discrete Steklov-Poincare operator $\mathscr{S}_{h}: W_{h} \rightarrow W_{h}^{\prime}$ by

$$
\left\langle\mathscr{S}_{h} q_{h}, \lambda_{h}\right\rangle:=\int_{\Omega_{f}} \sigma_{f}\left(\nabla u_{f}\right) \cdot \nabla \operatorname{Tr}_{h}^{-1} \lambda_{h} d x \quad, \quad \forall \lambda_{h} \in W_{h},
$$

where $u_{f}$ is the solution of the following Dirichlet problem:

$$
\left\{\begin{array}{c}
a_{f}\left(u_{f}, v_{f}\right):=\int_{\Omega_{f}} \sigma_{f}\left(\nabla u_{f}\right) \cdot \nabla v_{f} d x=0, \\
\forall v_{f} \in H_{0 h}^{1}\left(\Omega_{f}\right)=H_{h}^{1}\left(\Omega_{f}\right) \cap \operatorname{Ker}\left(\operatorname{Tr}_{h}\right), \\
\operatorname{Tr}_{h} u_{f}=q_{h} \quad \text { on } S .
\end{array}\right.
$$

Here, $\operatorname{Tr}_{h}^{-1} \lambda_{h}$ is any function in $H_{h}^{1}\left(\Omega_{f}\right)$ whose weak trace is equal to $\lambda_{h}$. We also introduce the following space:

$$
V_{i h}^{\text {ext }}=\left\{\left(v_{h}, v_{f}\right) \in V_{i h} \times H_{h}^{1}\left(\Omega_{f}\right), \operatorname{Tr}_{i h} v_{h}=\operatorname{Tr}_{h} v_{f} \text { on } S\right\} .
$$

With the above notation, we make the following assumptions.

Assumption 5.1. The bilinear form $a_{f}\left(u_{f}, v_{f}\right)$ satisfies the standard continuity and ellipticity conditions

$$
\left|a_{f}\left(u_{f}, v_{f}\right)\right| \leq C_{1}^{\prime}\left\|u_{f}\right\|_{1, \Omega_{f}}\left\|v_{f}\right\|_{1, \Omega_{f}} \quad, \quad \forall u_{f}, v_{f} \in H_{h}^{1}\left(\Omega_{f}\right),
$$




$$
a_{f}\left(u_{f}, u_{f}\right) \geq C_{2}^{\prime}\left\|u_{f}\right\|_{1, \Omega_{f}}^{2} \quad, \quad \forall u_{f} \in H_{h}^{1}\left(\Omega_{f}\right) .
$$

Assumption 5.2. There holds

$$
\inf _{q_{h} \in W_{h}} \sup _{v_{h} \in H_{h}^{1}\left(\Omega_{f}\right)}\left\{\frac{\int_{S} q_{h} v_{h} d a}{\left\|q_{h}\right\|_{L^{2}(S)}\left\|v_{h}\right\|_{L^{2}(S)}}\right\} \geq \beta>0 .
$$

We then have

Theorem 5.1. Assumption 4.1 is satisfied if Assumptions 5.1 and 5.2 are.

Proof. First, because of Assumption 5.2, Lemma 4.2 is applicable. Therefore, $\operatorname{Tr}_{h}$ is a continuous surjection from $H_{h}^{1}\left(\Omega_{f}\right)$ onto $W_{h}$, with a continuous inverse. Then, problem (33) can be written as

$$
\begin{aligned}
& a_{f}\left(u_{f}-\operatorname{Tr}_{h}^{-1} q_{h}, v_{f}\right)=-a_{f}\left(\operatorname{Tr}_{h}^{-1} q_{h}, v_{f}\right), \\
& \forall v_{f} \in H_{0 h}^{1}\left(\Omega_{f}\right),\left(u_{f}-\operatorname{Tr}_{h}^{-1} q_{h}\right) \in H_{0 h}^{1}\left(\Omega_{f}\right) .
\end{aligned}
$$

From Assumption 5.1, the above problem has a unique solution $u_{f}\left(q_{h}\right)$ satisfying

$$
\left\|u_{f}\right\|_{1, \Omega_{f}} \leq\left(\frac{C_{1}^{\prime}}{C_{2}^{\prime}}+1\right)\left\|\operatorname{Tr}_{h}^{-1} q_{h}\right\|_{1, \Omega_{f}} \leq C_{4}^{\prime}\left(\frac{C_{1}^{\prime}}{C_{2}^{\prime}}+1\right)\left\|q_{h}\right\|_{W} .
$$

By construction, we then have

$$
\begin{aligned}
\left|\left\langle\mathscr{S}_{h} q_{h}, \lambda_{h}\right\rangle\right| & =\left|a_{f}\left(u_{f}, \operatorname{Tr}_{h}^{-1} \lambda_{h}\right)\right| \\
& \leq C_{1}{ }^{\prime}\left\|u_{f}\right\|_{1, \Omega_{f}}\left\|\operatorname{Tr}_{h}^{-1} \lambda_{h}\right\|_{1, \Omega_{f}} \\
& \leq C^{\prime}\left\|q_{h}\right\|_{W}\left\|\lambda_{h}\right\|_{W} .
\end{aligned}
$$

In other words, $\mathscr{S}_{h}$ is a well-defined continuous operator from $H^{1 / 2}(S)$ in its dual. Moreover, since $\operatorname{Tr}_{h} u_{f}\left(q_{h}\right)=q_{h}$ by construction, we may also write

$$
\left\langle\mathscr{S}_{h} q_{h}, \hat{q}_{h}\right\rangle=a_{f}\left(u_{f}\left(q_{h}\right), u_{f}\left(\hat{q}_{h}\right)\right) .
$$

In this form, it is now clear that $\mathscr{S}_{h}$ is selfadjoint. To prove its coercivity, we rewrite this equality with $\hat{q}_{h}=q_{h}$, which implies

$$
\begin{aligned}
\left\langle\mathscr{S}_{h} q_{h}, q_{h}\right\rangle & =a_{f}\left(u_{f}\left(q_{h}\right), u_{f}\left(q_{h}\right)\right) \\
& \geq C_{2}^{\prime}\left\|u_{f}\left(q_{h}\right)\right\|_{1, \Omega_{f}}^{2} \\
& \geq C_{2}^{\prime} \frac{\left\|u_{f}\left(q_{h}\right)\right\|_{1, \Omega_{f}}^{2}}{\left\|\operatorname{Tr}_{h} u_{f}\right\|_{W}^{2}}\left\|\operatorname{Tr}_{h} u_{f}\right\|_{W}^{2} \\
& \geq \frac{C_{2}^{\prime}}{C_{3}^{\prime 2}}\left\|q_{h}\right\|_{W}^{2} .
\end{aligned}
$$

This is the last estimate needed to verify Assumption 4.1 .

We now turn to the practical solution of problem (11) in displacement when $\mathscr{S}_{h}$ is defined by (32). This turns out to be very simple, since we have 
Theorem 5.2. Under the above notation, the problem in displacement (11) takes the standard form

$$
\left\{\begin{array}{c}
a_{i}\left(u_{i}, w_{i}\right)+r a_{f}\left(u_{f}, w_{f}\right)=L_{i}\left(w_{i}\right)+\left\langle S_{h}\left(r q^{n-1}-\lambda_{i}^{n}\right), \operatorname{Tr}_{i h} w_{i}\right\rangle \\
\forall\left(w_{i}, w_{f}\right) \in V_{i h}^{\text {ext }},\left(u_{i}, u_{f}\right) \in V_{i h}^{\text {ext }} .
\end{array}\right.
$$

This is a coupled problem posed on the union of $\Omega_{i}$ and of the fictitious domain.

Proof. First, let $u_{i} \in V_{i h}$. Then, with $\operatorname{Tr}_{i h} u_{i} \in W_{h}$ we associate $u_{f}$ (extension of $\operatorname{Tr}_{i h} u_{i}$ on $\Omega_{f}$ ), solution of (33),

$$
\left\{\begin{array}{l}
a_{f}\left(u_{f}, v_{f}\right)=0, \forall v_{f} \in H_{0 h}^{1}\left(\Omega_{f}\right), \\
\operatorname{Tr}_{h} u_{f}=\operatorname{Tr}_{h} u_{i} \quad \text { on } S .
\end{array}\right.
$$

From the definition of the discrete Steklov-Poincare operator (32), we have

$$
\left\langle\mathscr{S}_{h} \operatorname{Tr}_{i h} u_{i}, \lambda_{h}\right\rangle=a_{f}\left(u_{f}, \operatorname{Tr}_{h}^{-1} \lambda_{h}\right) \quad, \quad \forall \lambda_{h} \in W_{h} .
$$

Moreover, by construction $\left(u_{i}, u_{f}\right) \in V_{i h}^{\text {ext }}$.

We now rewrite (11). Replacing the expression $\left\langle\mathscr{S}_{h} \operatorname{Tr}_{i h} u_{i}, w_{i}\right\rangle$ by $a_{f}\left(u_{f}, \operatorname{Tr}_{h}^{-1} \operatorname{Tr}_{i h} w_{i}\right)$, we get

$$
\begin{aligned}
& a_{i}\left(u_{i}, w_{i}\right)+r a_{f}\left(u_{f}, \operatorname{Tr}_{h}^{-1} \operatorname{Tr}_{i h} w_{i}\right) \\
& \quad=L_{i}\left(w_{i}\right)+\left\langle S_{h}\left(r q^{n-1}-\lambda_{i}^{n}\right), \operatorname{Tr}_{i h} w_{i}\right\rangle, \forall w_{i} \in V_{i h}, \\
& r a_{f}\left(u_{f}, w_{f}\right)=0, \forall w_{f} \in H_{0 h}^{1}\left(\Omega_{f}\right), \\
& \left(u_{i}, u_{f}\right) \in V_{i h}^{\mathrm{ext}} .
\end{aligned}
$$

If we add the two equalities and if we set $w=\left(w_{i}, \operatorname{Tr}_{h}^{-1} \operatorname{Tr}_{i h} w_{i}+w_{f}\right)$, we precisely get (35).

Conversely, from (35), we get the first line of the above system by setting $w=\left(w_{i}, \operatorname{Tr}_{h}^{-1} \operatorname{Tr}_{i h} w_{i}\right)$ and we get the second line by setting $w=\left(0, w_{f}\right)$ with $w_{f} \in H_{0 h}^{1}\left(\Omega_{f}\right)$. Altogether, this proves the desired equivalence result between (11) and (35).

But now, problem (35) is easy to solve. Indeed, if we use Lagrange multipliers to enforce the weak continuity $\operatorname{Tr}_{i h} u_{i}=\operatorname{Tr}_{h} u_{f}$ on the interface $S$ between $\Omega_{i}$ and $\Omega_{f}$, (35) takes the following form:

$$
\begin{aligned}
& a_{i}\left(u_{i}, w_{i}\right)+\left\langle p_{i h}, \operatorname{Tr}_{i h} w_{i}\right\rangle \\
& \quad=L\left(w_{i}\right)+\left\langle S_{h}\left(r q^{n-1}-\lambda_{i}^{n}\right), \operatorname{Tr}_{i h} w_{i}\right\rangle, \forall w_{i} \in V_{i h}, \\
& r a_{f}\left(u_{f}, w_{f}\right)-\left\langle p_{i h}, \operatorname{Tr}_{h} w_{f}\right\rangle=0, \forall w_{f} \in H_{h}^{1}\left(\Omega_{f}\right), \\
& \quad\left\langle\mu_{i h}, \operatorname{Tr}_{i h} u_{i}-\operatorname{Tr}_{h} u_{f}\right\rangle=0, \forall \mu_{i h} \in W_{h} .
\end{aligned}
$$

Moreover, a straightforward manipulation yields $p_{i h}=r \mathscr{S}_{h} \operatorname{Tr}_{i h} u_{i}^{n}$.

Problem (36)-(38) has the same structure as the global problem proposed in $\S 3$ of [18] and can therefore be solved by the dual conjugate gradient algorithm in $\S 3$ of [18]. 


\section{NUMERICAL RESULTS}

6.1. Generalities. In this section we describe some numerical results obtained with Algorithm (11)-(14). Our preconditioner is the discrete Steklov-Poincare operator of a fictitious domain $\Omega_{f}$, and the associated algorithm is compared with the unpreconditioned version $\left(\mathscr{S}_{h}=M_{W}^{2}\right)$. This comparison is done for various mesh sizes and various numbers of subdomains in the case of matching and nonmatching grids. The numerical implementation has been done within the MODULEF Finite Element library in a multi-element and multi-problem framework. For all experiments to be described below, the stopping criterion of Algorithm (11)-(14) was

$$
\frac{\left\|U^{n}-U^{n-1}\right\|_{2}}{\left\|U^{n}\right\|_{2}}<10^{-4} .
$$

In addition, the corresponding physical problem is the linear elasticity problem described in the introduction, with constitutive law

$$
\sigma=\frac{E \nu}{(\nu+1)(1-2 \nu)} \operatorname{Tr} \varepsilon \operatorname{Id}+\frac{E}{(\nu+1)} \varepsilon, \quad \varepsilon=\frac{1}{2}\left(\nabla u+\nabla u^{t}\right) .
$$

Here, $E$ and $\nu$ are respectively the Young modulus and the Poisson coefficient.

The domain $\Omega$ is a beam of section $0.5 \mathrm{~m} \times 0.2 \mathrm{~m}$ and length $1 \mathrm{~m}$ (see Figure 4). The beam is made of a quasi-incompressible material with $E=10^{11} \mathrm{MPa}$ and $\nu=0.49$ and is partitioned into second-order tetrahedral finite elements.

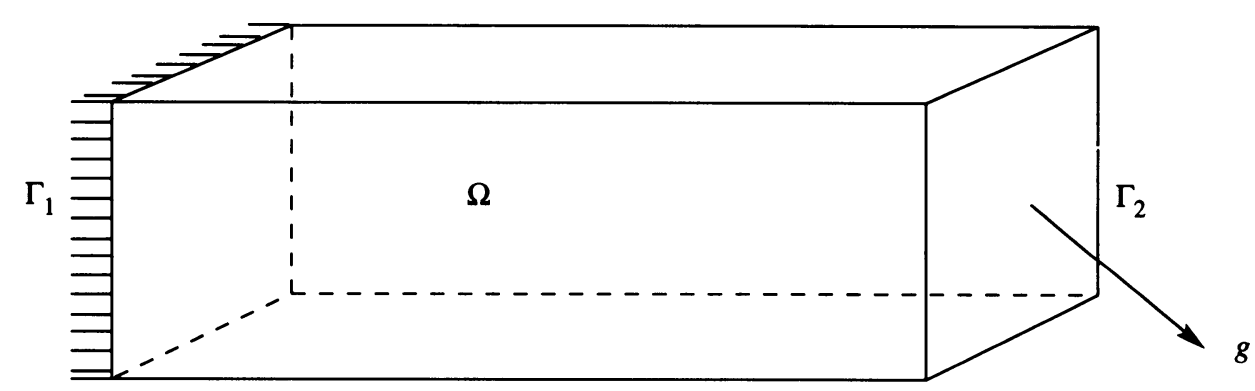

FIGURE 4. The physical configuration

Remark 6.1. The value $r_{\text {opt }}$ (optimal value of $r$ ) in the following tables was obtained by testing by hand different possible values. The lack of an automatic strategy for the choice of $r_{\mathrm{opt}}$ is a limitation of the algorithm.

\subsection{Test over the discretization step $h$.}

Example 6.1. First, we have tested the dependency on $h$ in the unpreconditioned version of Algorithm (11)-(14) $(\S 4.5)$ in the case where the beam has been sliced along its leading dimension into two domains of equal size. 
TABLE 1. Test over $h$ with the unpreconditioned version (matching grids)

d.o.f : degrees of freedom

\begin{tabular}{|c|c|c|c|c|c|}
\hline \hline$h^{-1}$ & $r / E$ & iter. & d.o.f in $\Omega_{1}$ & d.o.f in $\Omega_{2}$ & d.o.f in $S$ \\
\hline \multirow{2}{*}{23} & $0.1510^{4}$ & 144 & & & \\
& $0.18 \quad 10^{4}\left(r_{\text {opt }}\right)$ & 98 & 810 & 810 & 270 \\
& $0.2 \quad 10^{4}$ & 134 & & & \\
\hline \multirow{4}{*}{46} & $0.110^{4}$ & 260 & & & \\
& $0.510^{4}\left(r_{\text {opt }}\right)$ & 182 & 5049 & 5049 & 891 \\
& $10^{4}$ & 186 & & & \\
& $1.510^{4}$ & 272 & & & \\
\hline \hline
\end{tabular}

TABLE 2. Test over $h$ with the unpreconditioned version (nonmatching grids)

\begin{tabular}{|c|c|c|c|c|c|}
\hline$h^{-1}$ & $\overline{r / E}$ & iter. & d.o.f in $\Omega_{1}$ & d.o.f in $\Omega_{2}$ & d.o.f in $S$ \\
\hline 14 & $\begin{array}{c}0.13 \quad 10^{4} \\
0.15 \quad 10^{4}\left(r_{\text {opt }}\right) \\
0.2 \quad 10^{4}\end{array}$ & $\begin{array}{l}142 \\
120 \\
148\end{array}$ & 810 & 525 & 225 \\
\hline 28 & $\begin{array}{c}0.510^{4} \\
1.510^{4}\left(r_{\mathrm{opt}}\right) \\
210^{4}\end{array}$ & $\begin{array}{l}504 \\
400 \\
402\end{array}$ & 5049 & 3159 & 729 \\
\hline
\end{tabular}

Tables 1 and 2 show how the number of iterations and the optimal value of $r$ depend on the parameter $h$, roughly showing an $h^{-1}$ behavior. Moreover, the speed of convergence is very sensitive to the operator $\operatorname{Tr}_{h}^{-1}$; this explains the strong increase in the number of iterations for a finer mesh in the case of nonmatching grids.

Example 6.2. Now, we consider the same examples as above, but solved with the fictitious Schur preconditioner of $\S 5$. The fictitious domain $\Omega_{f}(0.1 \mathrm{~m} \times$ $0.5 \mathrm{~m} \times 0.2 \mathrm{~m}$ ) is applied on the interface $S$ and is fixed on $\Gamma_{D}^{f}$. It has the same constitutive material as the beam (Figure 5).

Tables 3 and 4 show that the preconditioned Algorithm (11)-(14) converges at a rate which is independent of $r$ and of the mesh size. Only a slight dependence on $h$ appears in the case of nonmatching grids. 


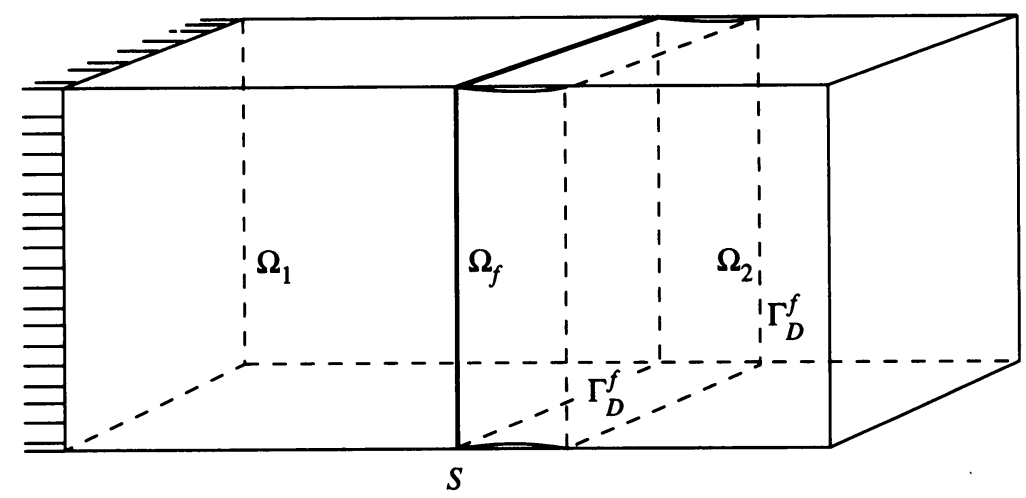

Figure 5. Decomposition in two subdomains

TABLE 3. Test over $h$ in the case of matching grids (fictitious domain)

\begin{tabular}{|c|c|c|c|c|c|}
\hline \hline step & $r$ & iter. & d.o.f in $\Omega_{1} \cup \Omega_{f}$ & d.o.f in $\Omega_{f} \cup \Omega_{2}$ & d.o.f in $S$ \\
\hline \multirow{4}{*}{$h$} & 0.6 & 64 & & & \\
& $0.5\left(r_{\text {opt }}\right)$ & 60 & 990 & 990 & 270 \\
& 0.4 & 74 & & & \\
\hline \multirow{3}{*}{$h / 2$} & 0.6 & 58 & & & \\
& $0.5\left(r_{\text {opt }}\right)$ & 56 & 5643 & 5643 & 891 \\
& 0.4 & 66 & & & \\
\hline
\end{tabular}

TABLE 4. Test over $h$ in the case of nonmatching grids (fictitious domain)

\begin{tabular}{|c|c|c|c|c|c|}
\hline \hline step & $r$ & iter. & d.o.f in $\Omega_{1} \cup \Omega_{f}$ & d.o.f in $\Omega_{f} \cup \Omega_{2}$ & d.o.f in $S$ \\
\hline \multirow{4}{*}{$h$} & 0.6 & 68 & & & \\
& $0.5\left(r_{\text {opt }}\right)$ & 64 & 960 & 675 & 225 \\
& 0.4 & 72 & & & \\
\hline \multirow{3}{*}{$h / 2$} & 0.6 & 86 & & & \\
& $0.5\left(r_{\text {opt }}\right)$ & 76 & 5535 & 3645 & 729 \\
& 0.4 & 96 & & & \\
\hline \hline
\end{tabular}

Example 6.3. Here, we consider the same beam as in Example 6.2, but decomposed into four geometrically identical subdomains (Figure 6). 


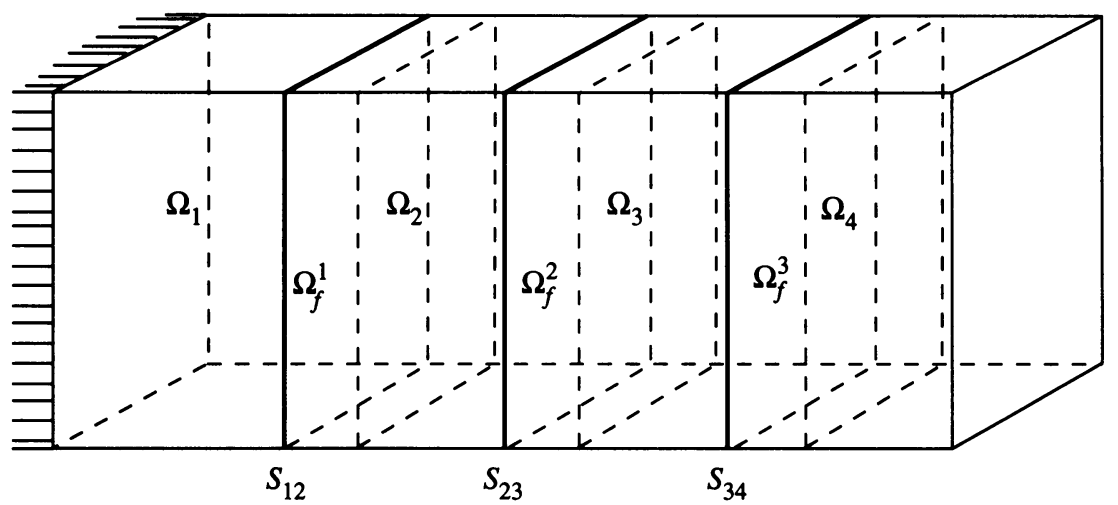

FIGURE 6. Decomposition in four subdomains

TABLE 5. Test over $h$ in the case of matching grids (fictitious domain)

\begin{tabular}{|c|c|c|c|c|c|c|c|}
\hline \hline step & $r$ & iter. & $\begin{array}{c}\text { d.o.f in } \\
\Omega_{1} \cup \Omega_{f}^{1}\end{array}$ & $\begin{array}{c}\text { d.o.f in } \\
\Omega_{2} \cup_{i=1}^{2} \Omega_{f}^{i}\end{array}$ & $\begin{array}{c}\text { d.o.f in } \\
\Omega_{3} \cup_{i=2}^{3} \Omega_{f}^{i}\end{array}$ & $\begin{array}{c}\text { d.o.f in } \\
\Omega_{f}^{3} \cup \Omega_{4}\end{array}$ & $\begin{array}{c}\text { d.o.f in } \\
S\end{array}$ \\
\hline$h$ & $\begin{array}{c}0.3 \\
0.4\left(r_{\text {opt }}\right) \\
0.5\end{array}$ & $\begin{array}{c}92 \\
78\end{array}$ & 630 & 810 & 810 & 630 & 810 \\
\hline$h / 2$ & $\begin{array}{c}0.3 \\
0.4\left(r_{\text {opt }}\right) \\
0.5\end{array}$ & $\begin{array}{c}94 \\
88\end{array}$ & 3267 & 3861 & 3861 & 3267 & 2673 \\
\hline \hline
\end{tabular}

TABLE 6. Test over $h$ in the case of nonmatching grids (fictitious domain)

\begin{tabular}{|c|c|c|c|c|c|c|c|}
\hline step & $r$ & iter. & $\begin{array}{l}\text { d.o.f in } \\
\Omega_{1} \cup \Omega_{f}^{1}\end{array}$ & $\begin{array}{c}\text { d.o.f in } \\
\Omega_{2} \cup_{i=1}^{2} \Omega_{f}^{i}\end{array}$ & $\begin{array}{c}\text { d.o.f in } \\
\Omega_{3} \cup_{i=2}^{3} \Omega_{f}^{i}\end{array}$ & $\begin{array}{l}\text { d.o.f in } \\
\Omega_{f}^{3} \cup \Omega_{4}\end{array}$ & $\begin{array}{c}\text { d.o.f in } \\
S\end{array}$ \\
\hline$h$ & $\begin{array}{c}0.25 \\
0.3\left(r_{\mathrm{opt}}\right) \\
0.35 \\
0.4\end{array}$ & $\begin{array}{c}118 \\
78 \\
90 \\
108\end{array}$ & 600 & 600 & 750 & 450 & 675 \\
\hline$h / 2$ & $\begin{array}{c}0.3 \\
0.35 \\
0.4\left(r_{\text {opt }}\right) \\
0.45\end{array}$ & $\begin{array}{l}120 \\
110 \\
104 \\
114\end{array}$ & 3159 & 2673 & 3645 & 2187 & 2187 \\
\hline
\end{tabular}

Tables 5 and 6 show that there is a slight increase in the number of iterations when we refine the mesh in the case of matching and nonmatching grids. The explanation may be the fact that we choose the same coefficient $r$ on each subdomain (it might be better to choose different $r$ on different subdomains). 
Compared to the unpreconditioned version, CPU times and residual histories (Figures 7 and 8) show that our preconditioner turns out to be preferable when dealing with fine grids. The CPU times obtained for the Schur version also include the time required for memory swapping, which is very large for a problem of this size run on a Sun Sparc 2 workstation .

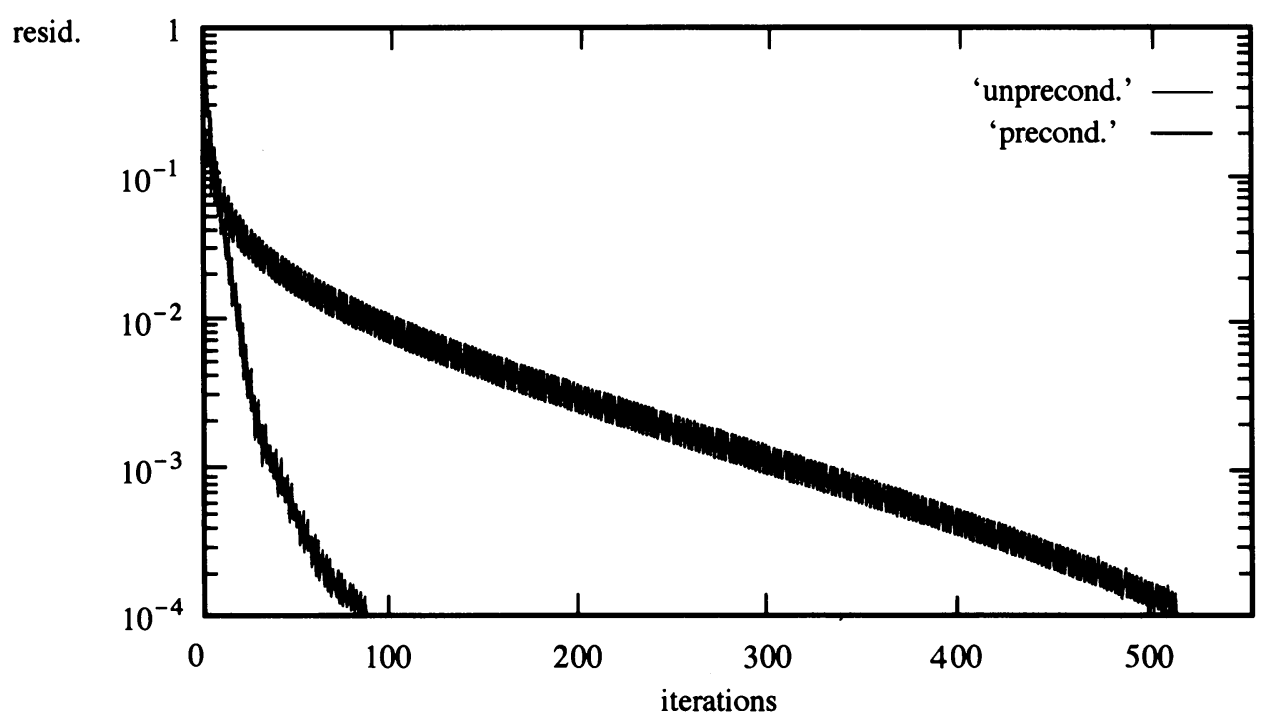

FIGURE 7. Residual against the iterations for a finer grid in the case of matching grids

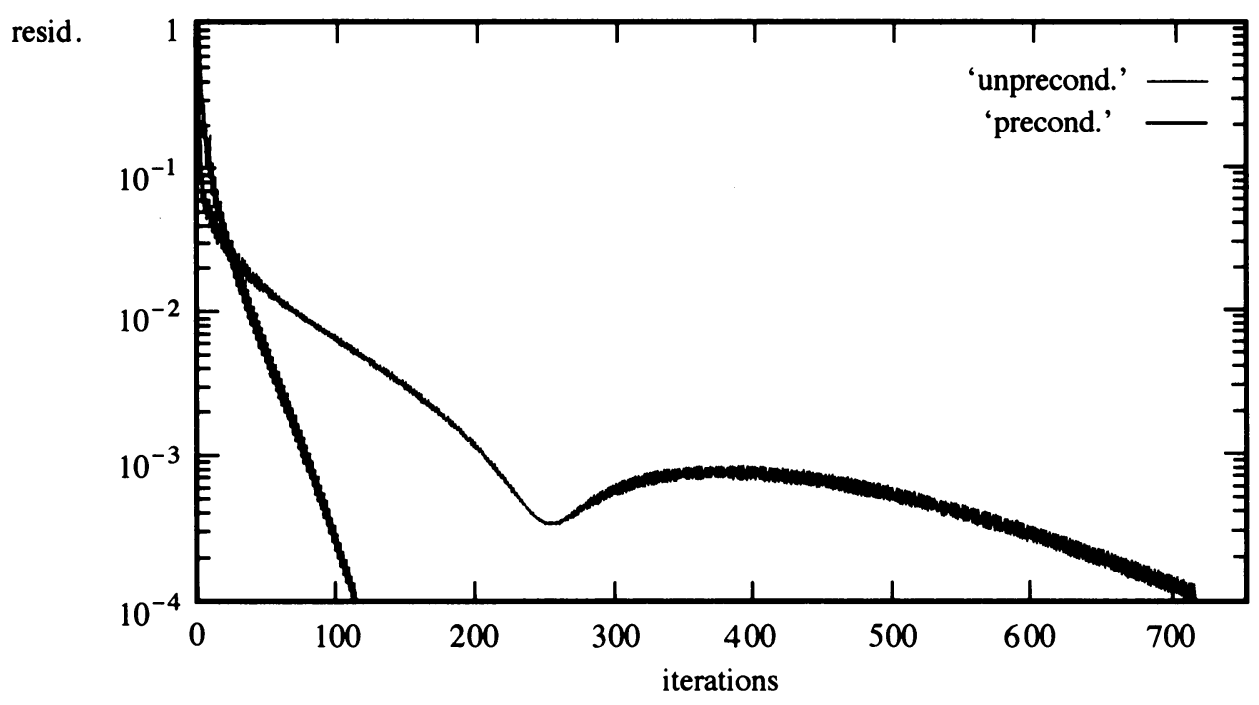

FIGURE 8. Residual against the iterations for a finer grid in the case of nonmatching grids 
TABLE 7. Comparison with the unpreconditioned version for a finer grid in the case of matching grids

\begin{tabular}{|c|c|c|c|}
\hline $\mathscr{S}_{h}$ & iter. & residual & total approximate time \\
\hline$M_{W}^{2}$ & 514 & $0.99310^{-4}$ & $2146 \mathrm{sec}$. \\
\hline fictitious Schur & 88 & $0.99510^{-4}$ & 1785 sec. \\
\hline
\end{tabular}

TABLE 8. Comparison with the unpreconditioned version for a finer grid in the case of nonmatching grids

\begin{tabular}{|c|c|c|c|}
\hline $\mathscr{S}_{h}$ & iter. & residual & total approximate time \\
\hline$M_{W}^{2}$ & 730 & $0.99310^{-4}$ & 2310 sec. \\
\hline fictitious Schur & 104 & $0.96310^{-4}$ & $2250 \mathrm{sec}$. \\
\hline
\end{tabular}

\subsection{Comparison with Neumann-Neumann preconditioner.}

Example 6.4. Here, our domain is a beam $(1 \mathrm{~m} \times 0.5 \mathrm{~m} \times 0.2 \mathrm{~m})$ consisting of parallel pencils. Two of them are made of a compressible material with $E_{m}=$ $1 \mathrm{MPa}$ and $\nu=0.31$, the third is made of a quasi-incompressible material with $E_{r}=10^{3} \mathrm{MPa}$ and $\nu=0.49$ (see Figure 9).

In Tables 9 and 10, the CPU time on a sequential machine for Algorithm (11)(14) with fictitious Schur preconditioner is compared to the CPU time for the Neumann-Neumann algorithm of [18] in the case of matching and nonmatching grids. The advantage of the second approach lies in the fact that it is less sensitive to the operator $\operatorname{Tr}_{h}^{-1}$ in the case of nonmatching grids. A second advantage of the second approach is that it does not require the a priori choice of a parameter $r_{\mathrm{opt}}$.

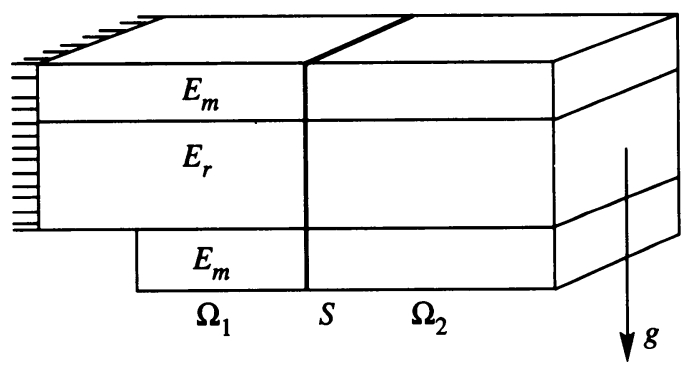

FIGURE 9 
TABLE 9. Comparison with the Neumann-Neumann preconditioner in the case of matching grids

\begin{tabular}{|l|c|c|}
\hline \hline & Neumann-Neumann & fictitious Schur \\
\hline d.o.f in $\Omega$ & 6102 & 7074 \\
assembly and factorization time & $760 \mathrm{sec}$. & $651 \mathrm{sec}$. \\
iterations & 66 & 74 \\
iterations time & $807 \mathrm{sec}$. & $765 \mathrm{sec}$. \\
\hline \hline total time & $1567 \mathrm{sec}$. & $1416 \mathrm{sec}$. \\
\hline \hline
\end{tabular}

TABLE 10. Comparison with the Neumann-Neumann preconditioner in the case of nonmatching grids

\begin{tabular}{|l|c|c|}
\hline \hline & Neumann-Neumann & fictitious Schur \\
\hline d.o.f in $\Omega$ & 8100 & 9072 \\
assembly and factorization time & $910 \mathrm{sec}$. & $691 \mathrm{sec}$. \\
iterations & 72 & 131 \\
iterations time & $960 \mathrm{sec}$. & $1444 \mathrm{sec}$. \\
\hline \hline total time & $1870 \mathrm{sec}$. & $2135 \mathrm{sec}$. \\
\hline
\end{tabular}

\section{CONCLUSION}

A Lagrangian formulation of a domain decomposed elasticity problem has been introduced and studied. For a small number of subdomains and very fine grids, this approach leads to efficient numerical algorithms, even in the case of nonmatching grids. Indeed, with the choice of adequate preconditioners such as the one introduced in $\S 5$, the method is proved to converge independently of the discretization step, which is confirmed by our numerical tests. Nevertheless, its practical implementation still faces the problem of the optimal choice of the regularization parameter $r$. Moreover, its convergence speed is only linear, and it does not involve any coarse grid solver. For these reasons, more classical algorithms based on preconditioned Schur complement methods might still be more competitive.

\section{APPENDIX}

Appendix 1. Proof of Lemma 4.1. By definition of $\operatorname{Tr}_{i h}$ (the $L^{2}$ projection operator from $V_{i h}$ into $\left.W_{h}\right)$, we have for any $v$ in $H^{1}(W)=\left\{v \in H^{1}(S), v=0\right.$ on $\left.\partial \Omega_{D} \cap \bar{S}\right\}$

$$
\left\|\operatorname{Tr}_{i h} v\right\|_{0, S} \leq\|v\|_{0, S} .
$$

We now denote by $r_{h}$ the Clement operator introduced in P. Clement [7] and described in F. Brezzi and M. Fortin ([6, p 105]). By construction, this 
operator satisfies

$$
\begin{gathered}
\left\|r_{h} v\right\|_{1, S} \leq C\|v\|_{1, S}, \\
\left\|r_{h} v-v\right\|_{0, s} \leq C h\|v\|_{1, s} .
\end{gathered}
$$

By setting $\operatorname{Tr}_{i h} v=r_{h} v+\operatorname{Tr}_{i h} v-r_{h} v$, we then have for any $v$ in $H^{1}(W)$

$$
\begin{aligned}
\left\|\operatorname{Tr}_{i h} v\right\|_{1, S} & \leq\left\|r_{h} v\right\|_{1, S}+\left\|\operatorname{Tr}_{i h} v-r_{h} v\right\|_{1, S} \\
& \leq\left\|r_{h} v\right\|_{1, S}+C h^{-1}\left\|\operatorname{Tr}_{i h} v-r_{h} v\right\|_{0, S} \\
& \leq\left\|r_{h} v\right\|_{1, S}+C h^{-1}\left(\left\|\operatorname{Tr}_{i h} v-v\right\|_{0, S}+\left\|v-r_{h} v\right\|_{0, S}\right) \\
& \leq\left\|r_{h} v\right\|_{1, S}+2 C h^{-1}\left\|v-r_{h} v\right\|_{0, S} \\
& \leq C\|v\|_{1, S} .
\end{aligned}
$$

By interpolation between $L^{2}(S)$ and $H^{1}(W)$, we have finally

$$
\left\|\operatorname{Tr}_{i h} v\right\|_{W} \leq C\|v\|_{W}
$$

A key point in the above proof is the inverse inequality $\left\|w_{h}\right\|_{1, s} \leq$ $C h^{-1}\left\|w_{h}\right\|_{0, s}$ used on $W_{h}$, which requires that the triangulation on $W_{h}$ must be uniformly regular.

If $\operatorname{Tr} V_{i h}$ is also built on a uniformly regular triangulation, then the same lemma holds also for the $L^{2}$ projection $\Pi_{i h}$ onto $\operatorname{Tr} V_{i h}$.

Appendix 2. Proof of Lemma 4.2. From Assumption 4.2, there exists a mapping $B_{i}^{-1}$ from $W_{h}$ into $\operatorname{Tr} V_{i h}$ such that

$$
\begin{gathered}
\int_{S} B_{i}^{-1} w_{h} \mu_{h} d a=\int_{S} w_{h} \mu_{h} d a, \forall \mu_{h} \in W_{h}, \\
\left\|B_{i}^{-1} w_{h}\right\|_{0, S} \leq \frac{1}{\beta}\left\|w_{h}\right\|_{0, S} .
\end{gathered}
$$

By extension, we will also denote by $B_{i}^{-1} w$ the action of $B_{i}^{-1}$ on the $L^{2}$ projection of $w$ on $W_{h}$ for any $w \in L^{2}(S)$. We now define

$$
\hat{w}_{i h}=\Pi_{i h} w_{i h}+B_{i}^{-1}\left(w_{i h}-\Pi_{i h} w_{i h}\right) \in \operatorname{Tr} V_{i h}, \quad \forall w_{i h} \in W_{h},
$$

with $\Pi_{i h}$ the $L^{2}$ projection operator onto $\operatorname{Tr} V_{i h}$. By construction, we have $\operatorname{Tr}_{i h} \hat{w}_{i h}=w_{i h}$ and

$$
\left\|\hat{w}_{i h}\right\|_{1, S} \leq\left\|\Pi_{i h} w_{i h}\right\|_{1, S}+\left\|B_{i}^{-1}\left(w_{i h}-\Pi_{i h} w_{i h}\right)\right\|_{1, S} .
$$

But, since the triangulation is uniform on $V_{i h}$, we have

$$
\left\|B_{i}^{-1}\left(w_{i h}-\Pi_{i h} w_{i h}\right)\right\|_{1, S} \leq \frac{C}{h}\left\|B_{i}^{-1}\left(w_{i h}-\Pi_{i h} w_{i h}\right)\right\|_{0, s}
$$


Hence, it follows from Appendix 1 that $\left\|\hat{w}_{i h}\right\|_{1, S}$ satisfies

$$
\begin{aligned}
\left\|\hat{w}_{i h}\right\|_{1, S} & \leq\left\|\Pi_{i h} w_{i h}\right\|_{1, s}+\frac{C}{h}\left\|B_{i}^{-1}\left(w_{i h}-\Pi_{i h} w_{i h}\right)\right\|_{0, S} \\
& \leq C\left\|w_{h}\right\|_{1, S}+\frac{C}{\beta h}\left\|w_{i h}-\Pi_{i h} w_{i h}\right\|_{0, s} \\
& \leq C\left\|w_{h}\right\|_{1, s}+\frac{C}{\beta h}\left\|w_{i h}-r_{h} w_{i h}\right\|_{0, s} \\
& \leq\left(C+\frac{C C^{\prime} h}{\beta h}\right)\left\|w_{i h}\right\|_{1, S} \\
& \leq C\left(1+\frac{C^{\prime}}{\beta}\right)\left\|w_{i h}\right\|_{1, S} .
\end{aligned}
$$

By interpolation between $H^{1}(W)$ and $L^{2}(S)$, we then obtain

$$
\left\|\hat{w}_{i h}\right\|_{W} \leq C\left\|w_{i h}\right\|_{W} .
$$

We now simply define $\operatorname{Tr}_{i h}^{-1} w_{h}$ as the solution of the Dirichlet problem

$$
\begin{aligned}
& \int \nabla \operatorname{Tr}_{i h}^{-1} w_{h} \cdot \nabla v_{h} d x=0, \forall v_{h} \in V_{i h}^{0}, \operatorname{Tr}_{i h}^{-1} w_{h} \in V_{i h}, \\
& \operatorname{Tr}_{i h}^{-1} w_{h}=\hat{w}_{i h} \text { on } S,
\end{aligned}
$$

posed on the space

$$
V_{i h}^{0}=\left\{v_{i h} \in V_{i h}, v_{i h \mid S}=0\right\} .
$$

\section{BIBLIOGRAPHY}

1. C. Bernardi, Y. Maday, and T. Patera, A new nonconforming approach to domain decomposition: the mortar element method, Nonlinear Partial Differential Equations and their Applications, Collège de France Seminar, Pitman, 1990; also report 89027 of Laboratoire d'Analyse Numérique, Univ. Paris 6.

2. P.E. Bjørstad and O. B. Widlund, Iterative methods for the solution of elliptic problems on regions partitioned into substructures, SIAM J. Numer. Anal. 23 (1986), 1097-1120.

3. J.F. Bourgat, R. Glowinski, P. Le Tallec, and M. Vidrascu, Variational formulation and algorithm for trace operator in domain decomposition calculations, Proc. 2nd Internat. Sympos. on Domain Decomposition Methods (Los Angeles, CA, January 1988), SIAM, Philadelphia, PA, 1989.

4. J.H. Bramble, J.E. Pasciak, and A.H. Schatz, An iterative method for elliptic problems on regions partitioned into substructures, Math. Comp. 46 (1986), 361-369.

5. Comp. 53 (1989), 1-24.

6. F. Brezzi and M. Fortin, Mixed and hybrid finite element methods, Springer-Verlag, New York, 1991.

7. P. Clement, Approximation by finite element functions using local regularization, R.A.I.R.O. Anal. Numér. 9 (1974), 77-84.

8. Y.H. De Roeck, P. Le Tallec, and M. Vidrascu, Domain decomposition methods for large linearly elliptic three dimensional problems, J. Comput. Appl. Math. 34 (1991), 93-117.

9. M. Dryja, B. Smith, and O. Widlund, Schwarz analysis of iterative substructuring algorithms for elliptic problems in three dimensions, SIAM J. Numer. Anal. 31 (1994), 1662-1694. 
10. M. Dryja and O. Widlund, Towards a unified theory of domain decomposition algorithms for elliptic problems, Proc. Third Internat. Sympos. on Domain Decomposition Methods (Houston), SIAM, Philadelphia, PA, 1990.

11. C. Farhat and F.X. Roux, Implicit parallel processing in structural mechanics, Computational Mechanics Advance (J. T. Oden, ed.), Vol. 2, North-Holland, Amsterdam, 1994, pp 1-124.

12. M. Fortin and R. Glowinski, Augmented Lagrangian methods, North-Holland, Amsterdam, 1983.

13. D. Gabay, Application of the methods of multipliers to variational inequalities (in [11]).

14. V. Girault and P.A. Raviart, Finite element methods for Navier-Stokes equations. Theory and algorithms, Springer-Verlag, Berlin, Heidelberg, New York, Tokyo, 1986.

15. E. Givois, Ph.D. Thesis, Univ. Paris Dauphine, Paris, 1992 (In French) .

16. R. Glowinski and P. Le Tallec, Augmented Lagrangian and operator splitting methods in nonlinear mechanics, SIAM, Philadelphia, PA, 1989.

17. P. Le Tallec, Domain decomposition method in computational mechanics, Computational Mechanics Advance (J. T. Oden, ed.), Vol. 1, North-Holland, Amsterdam, 1994.

18. P. Le Tallec and T. Sassi, Domain decomposition with nonmatching grids: Schur complement approach, Cahiers de mathématiques de la décision, n 0 9323, CEREMADE, Univ. Paris Dauphine, 1993.

19. P. Le Tallec, T. Sassi, and M. Vidrascu, Three-dimensional domain decomposition methods with nonmatching grids and unstructured coarse solvers, Proc. Seventh Internat. Sympos. on Domain Decomposition Methods, (D. Keyes and J. Xu, eds.), Contemp. Math., vol. 180, Amer. Math. Soc., Providence, RI, 1994, pp. 61-74.

20. P.L.Lions, On the Schwarz alternating method III: $A$ variant for nonoverlapping subdomains, In same proceedings as [10].

21. P.L. Lions and B. Mercier, Splitting algorithms for the sum of two nonlinear operators, SIAM J. Numer. Anal. 16 (1979), 964-979 .

22. S.V. Nepomnyaschikh, Mesh theorems on traces, normalizations of function traces and their inversion, Soviet J. Numer. Anal. Math. Modelling 6 (1991), 223-242.

Ceremade, Université Paris-Dauphine, Place du Maréchal de lattre de Tassigny, 75775 Paris Cedex 16, France

E-mail address: patrick.letallec@inria.fr

sassi@mathinsa.insa-lyon.fr 